\title{
Time Synchronization and Ranging in OFDM Systems Using Time-Reversal
}

\author{
Traian E. Abrudan, Member, IEEE, Azadeh Haghparast, and Visa Koivunen, Fellow, IEEE
}

\begin{abstract}
In this paper, we propose a new two-way time synchronization (TS) method for orthogonal frequency division multiplexing (OFDM)-based wireless systems. It relies on the time-reversal (TR) technique to remove the effect of the channel phase after the signal round-trip. Thus, the method is named TR-TS. TR technique yields a linear phase rotation across subcarriers, regardless whether the channel is minimum, maximum, or mixed phase. This phase is proportional to the difference of the OFDM symbol-timing synchronization errors at the two receivers. Thus, the clock-offset between the radios is determined using the local reception times and the estimated linear phase resulting from the TR technique. A reliable low-complexity algorithm called fast Fourier transform-weighted least-squares is proposed to estimate the linear phase slope, and its meansquare error is compared with the Cramér-Rao Lower Bound (CRLB). The results show that the proposed algorithm attains the CRLB at low SNR, even when a single OFDM symbol is used. The OFDM packets are time-stamped at the medium access control layer, which allows eliminating errors caused by different and varying delays in the internal lower level processing of the data. Hence, an accurate estimate of the clock-offset is obtained. The impact of various nonidealities on the proposed algorithm is also studied. In addition, we propose a ranging method that employs the novel TS method and a first-path delay estimation technique. The performance of the proposed methods is studied in simulations, as well as using real-world measured channels. The results show that the proposed methods can be successfully applied in low to moderate mobility scenarios such as indoors despite harsh multipath, since they rely on channel reciprocity.
\end{abstract}

Index Terms-Clocks, distance measurement, orthogonal frequency division multiplexing (OFDM), radio communication, synchronization.

\section{INTRODUCTION}

$\mathbf{I}$ N MANY wireless applications, a precise common time reference is required. The most common applications include: time ordering of events in distributed systems [2], networked measurement and control systems [3], [4] where readings from multiple sensors need to be synchronized, industrial automation, and real-time data fusion in wireless sensor networks (WSNs) [5], in general. Among communication applications, the most notable are time synchronization (TS) among

Manuscript received October 15, 2012; revised January 23, 2013; accepted March 16, 2013. Date of publication September 5, 2013; date of current version November 6, 2013. This work was supported in part by Nokia Research Center, WILATI+ Project, and the Graduate School in Electronics, Telecommunications, and Automation. The Associate Editor coordinating the review process was Dr. Dario Petri.

T. E. Abrudan is with the Instituto de Telecomunicações, Faculdade de Engenharia da Universidade do Porto, Porto 4200-465, Portugal (e-mail: tabrudan@fe.up.pt).

A. Haghparast and V. Koivunen are with Aalto University, SMARAD CoE, Espoo, PO. Box. 13000, 00076-FIN, Finland (e-mail: visa.koivunen@ aalto.fi). Digital Object Identifier 10.1109/TIM.2013.2272840 base stations [6], intervehicle synchronization in vehicular ad hoc networks [7], as well as cooperative communications, e.g., coherent space-time coding [8] and distributed transmit beamforming [9], when band-pass signals are employed. A reliable time reference also enables time-slotted medium access control (MAC) protocols for cognitive radios [10], coordinated adaptive sleeping in energy-limited devices [11], etc. Furthermore, precise TS may be used for wireless indoor positioning [12], which itself enables a multitude of other applications such as navigation, location-based services, as well as first-responder applications. Since most of the current and emerging wireless communication standards use orthogonal frequency division multiplexing (OFDM), it makes sense to develop localization and radar techniques based on OFDM waveforms. Moreover, [13] proves in terms of Cramér-Rao Lower Bound (CRLB) that ranging using OFDM waveform outperforms the pseudonoisebased ranging, at equal energy and bandwidth. Using the existing wireless network technologies, such as wireless local area networks (WLANs), or long-term evolution (LTE), for localization purposes has the advantage that no additional infrastructure is required to provide the users with indoor positioning. OFDM is also employed in the IEEE 802.11p vehicle-to-vehicle communication standard.

In this paper, we introduce a novel TS method for OFDMbased wireless systems using time-reversal (TR) technique [14]. The new TR-TS method will be referred to as TR-TS method in the rest of this paper. In addition, based on the proposed synchronization method, a novel ranging method is introduced. The TR-TS method and the corresponding ranging method are based on the round-trip time-of-flight of the OFDM signal and remote clock reading. Both methods involve a pair of wireless transceivers and no centralized control, hence they can be used in distributed systems.

The main contributions of this paper are listed as follows.

1) We propose a TS method that employs the TR technique to estimate the clock-offset between two radios. TR technique exploits the reciprocity of the channel to remove the effects of the channel phase after the signal round-trip. A linear phase rotation across OFDM subcarriers remains where the slope is proportional to the difference of symbol-timing errors at the two radios. This phase rotation can be accurately estimated and the clock-offset can be corrected. The proposed TR-TS method can deal with demanding non-line-of-sight (NLOS) multipath propagation environments such as the indoor channels. 
2) With the fast Fourier transform (FFT) and weighted least-squares (WLS), a computationally efficient FFTWLS algorithm is derived to estimate the phase parameter obtained in the TR technique. CRLB is established to evaluate the performance of the proposed algorithm. Simulations show that the mean-square error (MSE) corresponding to the FFT-WLS attains the CRLB at low SNR values, even on using a single observation vector.

3) With the proposed TR-TS method, a novel ranging method is introduced. An additional time-of-arrival (ToA) estimation technique is employed to estimate the distance between two OFDM radios. In this paper, a matrix pencil method is used to estimate the delay corresponding to the first arrival path [15]-[17].

4) The impact of various nonidealities on the proposed methods are studied: message delivery delays, channel nonstationarity, clock drifts, and carrier frequency offsets. A method for correcting the clock drifts is also proposed. Real-world indoor wireless channels are used to validate the performance of the proposed TS and ranging methods. The results show that the proposed methods are applicable in low to moderate mobility scenarios and severe multipath since they rely on channel reciprocity.

Achieving and maintaining precise TS in wireless networks is a challenging task because of inaccurate clocks, unknown propagation delays, as well as random delays at different layers of the protocol stack. A major source of error in many clock-synchronization methods is the random delay in the message transfer between two radios. The latency of the message delivery was first characterized in [18] as being composed of four distinct factors: Send Time, Access Time, Propagation Time, and Receive Time. In [19], it is demonstrated that the processing delay at the radios can be considered deterministic by time-stamping the packets at the MAC layer. This procedure, also adopted in this paper, eliminates the errors caused by different varying delays in the internal lower level processing in the transceivers such as packet construction/retrieval, operating system interrupts, queuing time at the MAC layer, etc.

Many of the existing TS methods neglect the propagation delay between the radios, because a sufficient timing accuracy is obtained for the application at hand, for example, ordering events in WSNs [2], [5]. This is definitely not acceptable in ranging systems, where the propagation delay is the crucial parameter of interest. For spatially localized measurement and control systems, the generic standardized IEEE 1588 Precision Clock Synchronization Protocol [3] enables synchronization accuracy and precision in the microsecond and submicrosecond range [20]. In this paper, we propose a clock synchronization approach for mobile entities that use OFDM waveforms. The problem of precise time transfer over a wireless communication link has also been addressed recently in [21]. The approach in [21] involves a remote transponder that distributes the clock to fixed sites separated by a precisely known distance, as well as additional hardware. In our approach, the two wireless terminals are mobile and no additional hardware in required, apart from the OFDM transceiver itself. There are various clock-synchronization algorithms in the literature, and their simpler form, TS algorithms $^{1}$ [5], [19], [22]-[28]. A survey of different distributed synchronization techniques in wireless networks is provided in [29]. To the best of our knowledge, matching the reference points for time-stamping at the two radios has not been considered in the TS mechanisms. The reason might be that most of the existing clock-synchronization methods have been developed for sensor networks applications, where often a coarser synchronization is sufficient, or for ultrawideband (UWB) systems [30], [31], where the large bandwidth of the signal provides the system with high time resolution. However, in narrowband OFDM-based systems, symbol-timing offset causes a large error in the estimated distance. One might think that this problem can be addressed through some of the existing symbol-timing synchronization methods, such as SchmidlCox algorithm [32] or Minn algorithm [33], [34]-[36]. However, due to severe multipath propagation and noise, there is no guarantee that the OFDM packets are time-stamped at exactly the same sampling instants at the two radios. In [37], it is explained that NLOS propagation is a limiting factor, which yields an additional bias to the timing measurements regardless of symbol-timing synchronization method. This problem is addressed this paper by using the TR technique. Therefore, there is no need for complicated symbol-synchronization algorithms, while a coarse one is sufficient.

Over the past few years, many ranging and localization methods have been proposed [12], [30], [38]-[40]. One of the most appealing ranging methods that can be applied to OFDM systems is based on super-resolution ToA estimation [41]-[43]. However, the main challenge in such methods is the accurate TS among the wireless terminals. This is in contrast to the UWB systems [30], [31], [44], where the high time resolution makes the accurate TS easier to achieve compared with systems with lower bandwidths, such as WLAN or LTE. [45] provides an analysis of super-resolution methods for timeof-flight estimation and shows that even in UWB scenarios, the undetected direct path is a performance limiting factor. Because of the OFDM symbol-timing synchronization errors, the reference points may be different at the two radios, which leads to error in clock-offset estimation, and consequently causes bias in the range or location estimates. For instance, in the case of IEEE 802.11/a standard, where the bandwidth is $20 \mathrm{MHz}$, a timing error of a single sampling period at the Nyquist rate will cause a range error of $15 \mathrm{~m}$. The idea of OFDM ranging using TR technique was introduced in our earlier work [1], where only integer delays were considered. In this paper, we propose a new algorithm that significantly improves the ranging performance considering fractional delays. Unlike our previous work [1], the present ranging approach relies on the new clock synchronization method proposed in this paper and remote clock reading. The impact of various nonidealities that were ignored in [1] is also considered. Furthermore, the algorithm performance was evaluated in simulations and using real-world measurements.

\footnotetext{
${ }^{1} \mathrm{TS}$ of two clocks means to set their time at a particular time instant to be the same. This is different from clock-synchronization in which the clocks are synchronized in both frequency and time.
} 
The rest of this paper is organized as follows. The OFDM system model is presented in Section II. The proposed TRTS method is described in Section III. This section contains the two-way TS protocol, as well as the TR technique for eliminating the OFDM symbol-timing errors. The proposed FFT-WLS approach for estimating the phase rotation resulting from the TR technique is derived in Section IV. Then, MSE corresponding to the proposed FFT-WLS and to the maximum likelihood (ML) estimator is compared with the CRLB. A ranging method using the proposed TR-TS is introduced in Section V. Section VI deals with the practical aspects of the problem at hand such as message delivery delays, channel reciprocity, clock drifts, and carrier frequency offsets. A method for correcting the clock drifts is also proposed. Finally, the performance of the proposed methods is studied in Section VII using simulations and real-world channel measurements. Section VIII concludes this paper. Additional derivations are provided in Appendix.

\section{OFDM System MODEL}

Let us consider two wireless terminals called Radios A and $B$ that have both a transmitter and a receiver. The terminals use an OFDM transmission with $N$ subcarriers, and a cyclic prefix $(\mathrm{CP})$ of length $L$. The employed sampling period is $T_{s}=T /(N+L)$, where $T$ represents the duration of the entire OFDM symbol. Let $\mathcal{X}_{n}$ be the constellation data point transmitted by Radio A, on the $n$th subcarrier. When noise only is present ${ }^{2}$ the frequency-domain OFDM data point received at Radio $\mathrm{B}$, on the $n$th subcarrier is

$$
\mathcal{Y}_{n}=\mathcal{H}_{n} \mathcal{X}_{n}+\mathcal{W}_{n}, \quad \text { for } n \in \mathbb{A}
$$

where $\mathcal{H}_{n}$ and $\mathcal{W}_{n}$ are the frequency response of the channel and the additive Gaussian noise on the $n$th subcarrier, respectively. $\mathbb{A}$ denotes the set of indices corresponding to the $2 N_{\mathrm{a}}$ active OFDM subcarriers, that are numbered as $\left\{-N_{\mathrm{a}}, \ldots,-1,+1, \ldots,+N_{\mathrm{a}}\right\}$ (the middle carrier is inactive).

\section{Proposed TR-TS Method}

In this section, we propose a TS method on the basis of the TR technique. The aim is to estimate the clock-offset between two OFDM wireless transceivers, namely, Radios A and B and synchronize them on demand. Therefore, the proposed method is suitable for distributed systems. The following assumptions are made

1) There is a bidirectional link between Radios A and B. The two radios operate on the same frequency band in both directions, using OFDM.

2) The channel between the two radios, including the pulseshaping filters at both transmitter and receiver remains reciprocal during the round-trip time-of-flight of the signal.

3) The clock readings are performed at the MAC layer. Therefore, the nondeterministic message delays are eliminated.

\footnotetext{
${ }^{2}$ Other nonidealities will be considered in detail later.
}

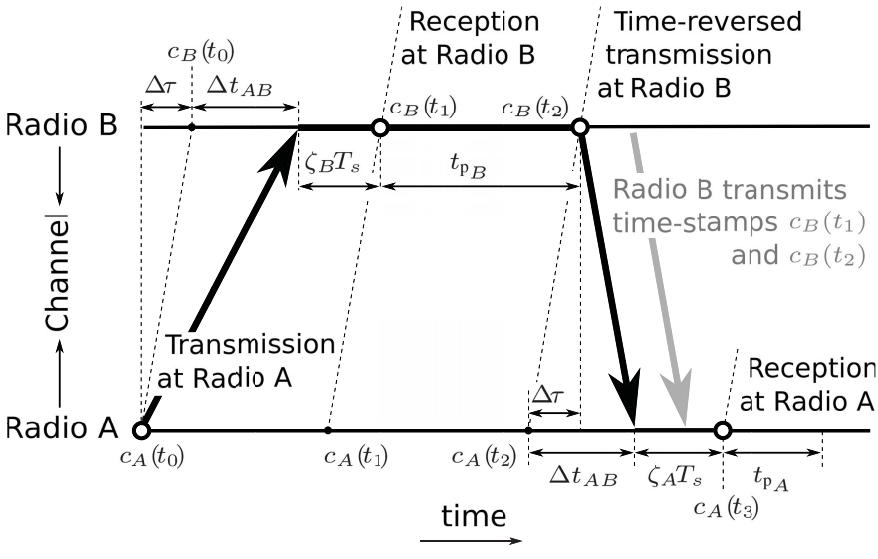

Fig. 1. Setup of the TR-TS method for estimating the clock-offset $\Delta \tau$ between Radios A and B. $c_{A}($.$) and c_{B}($.$) denote the clock values at Radios A$ and $\mathrm{B}$, respectively, and they are related by $c_{B}(t)=c_{A}(t)+\Delta \tau$. The propagation delay from one radio to the other radio is $\Delta t_{A B}$. Two time-stamps are transmitted from Radio B to Radio A. The processing delay at Radios A and B are $t_{\mathrm{p}_{A}}$ and $t_{\mathrm{p}_{B}}$, respectively. $\zeta_{A}$ and $\zeta_{B}$ represent the OFDM symboltiming errors at Radios $\mathrm{A}$ and $\mathrm{B}$, respectively.

The above assumptions are justified in Section VI. Let us denote the clock values of Radios A and B at the time instant $t$ by $c_{A}(t)$ and $c_{B}(t)$, respectively. The relation between $c_{A}(t)$ and $c_{B}(t)$ can be written as

$$
c_{B}(t)=c_{A}(t)+\Delta \tau
$$

where $\Delta \tau$ is the clock-offset between the two radios.

\section{A. Two-Way TS Protocol}

Fig. 1 shows the setup of the proposed TR-TS method. The TS protocol has the following steps.

1) Radio A initiates the TS procedure by transmitting a request message to Radio $\mathrm{B}$, at time instant $t_{0}$. The time indicated by the clock of Radio A at the start of transmission is denoted by $c_{A}\left(t_{0}\right)$.

2) The message experiences a propagation delay $\Delta t_{A B}$ on the forward channel and is received at Radio B with a timing error of $\zeta_{B}$ sampling periods $T_{S}$, at time instant $t_{1}$. The reception time indicated by the clock of Radio $\mathrm{B}$ is denoted by $c_{B}\left(t_{1}\right)$, and may be written as

$$
c_{B}\left(t_{1}\right)=c_{A}\left(t_{0}\right)+\Delta \tau+\Delta t_{A B}+\zeta_{B} T_{s} .
$$

3) A message containing the time-reversed version of the received OFDM signal is then transmitted back to Radio $\mathrm{A}$ at time instant $t_{2}$. This time is indicated by the clock of Radio B as $c_{B}\left(t_{2}\right)$. In addition, the reception timestamp $c_{B}\left(t_{1}\right)$ and the transmission time-stamp $c_{B}\left(t_{2}\right)$ are transmitted to Radio A. Note that $t_{\mathrm{p}_{B}}=\left[c_{B}\left(t_{2}\right)-c_{B}\left(t_{1}\right)\right]$ is the processing delay at Radio B. This delay could be fixed such that is known by Radio A. In that case, the time-stamp $c_{B}\left(t_{1}\right)$ solely would be sufficient for the clock-offset estimation. However, in our approach, we choose to transmit a second time-stamp $c_{B}\left(t_{2}\right)$ for two reasons. First, there are less strict time constraints on the processing delay. Second, and more importantly, this approach allows for clock drift estimation. If there is 
no drift between the two clocks $c_{A}$ and $c_{B}$, the time durations $\left[c_{B}\left(t_{2}\right)-c_{B}\left(t_{1}\right)\right]$ and $\left[c_{A}\left(t_{2}\right)-c_{A}\left(t_{1}\right)\right]$ will be equal (see Fig. 1).

4) Once again the message experiences a propagation delay $\Delta t_{A B}$ on the return channel and is received at Radio $\mathrm{A}$ at time instant $t_{3}$. This time is stamped by the clock of Radio $\mathrm{A}$ as $c_{A}\left(t_{3}\right)$. Therefore, after the round-trip, we have (see Fig. 1)

$$
c_{A}\left(t_{3}\right)=c_{B}\left(t_{2}\right)-\Delta \tau+\Delta t_{A B}+\zeta_{A} T_{S}
$$

where $\zeta_{A}$ is the symbol-timing error at Radio A.

5) Subtracting (3) from (4) allows for eliminating the unknown propagation time $\Delta t_{A B}$, to obtain the clockoffset between Radios A and B

$$
\begin{aligned}
\widehat{\Delta \tau}=\frac{1}{2}( & {\left[c_{B}\left(t_{1}\right)-c_{A}\left(t_{0}\right)\right]-\left[c_{A}\left(t_{3}\right)-c_{B}\left(t_{2}\right)\right] } \\
& \left.+\hat{\delta} T_{s}\right) .
\end{aligned}
$$

As we may notice in (5), all the clock values $c_{A}($.$) and$ $c_{B}($.$) are known at Radio A. The only unknown quantity$ is $\delta=\left(\zeta_{A}-\zeta_{B}\right)$, i.e., the difference between timing errors at Radios A and B, respectively. Its estimate $\hat{\delta}$ is obtained by employing the TR technique introduced in this paper. The equations describing the TR technique are explained in detail in the following subsection.

\section{B. TR Technique}

In this section, the key role of the TR technique is explained in detail. The proposed TS method requires that the reference points at which the clock readings need to be performed are accurately determined. In this paper, the reference point for time-stamping is chosen to be the first data sample (after the CP) of the first OFDM symbol in the packet. Because of severe multipath propagation and noise, the detection of the exact reference points at the two OFDM transceivers is subject to symbol-timing synchronization errors, regardless of the symbol-timing synchronization algorithm [37]. The OFDM symbol timing information is contained in the last term in (5). To obtain a precise timing, it is required to go beyond the sampling time resolution, i.e., consider fractional timing offsets that correspond to noninteger $\zeta_{A}$, and $\zeta_{B}$. The TR technique allows for a precise estimation of this timing information regardless of the wireless channel.

In the TR technique, the signal received from an unknown channel is reversed in time, energy normalized, and then retransmitted through the channel. Therefore, all the computations take place at one radio and the second radio merely performs TR. After the TR operation, the round-trip equivalent channel frequency response is obtained by multiplying the frequency response of the one-way channel by its complexconjugate. Therefore, the channel acts as a matched filter to itself. In time domain, this corresponds to the convolution of the channel impulse response with its time-reversed complexconjugate version, thus achieving an excellent time focusing of the signal [14], [46]-[48]. If no timing errors are present, the channel phase vanishes across all OFDM subcarriers resulting into a real-valued channel. Fig. 2 shows the frequency

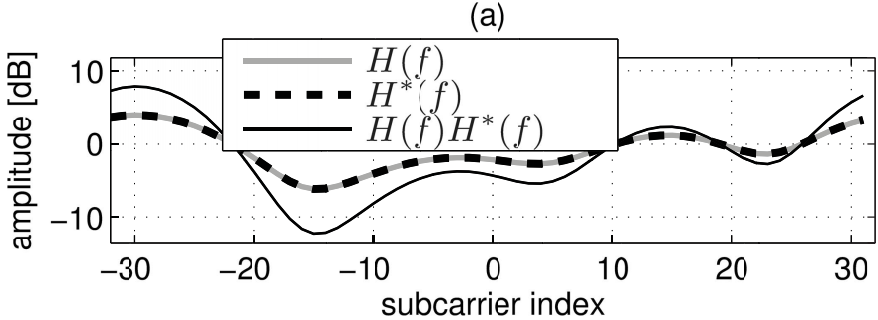

(b)

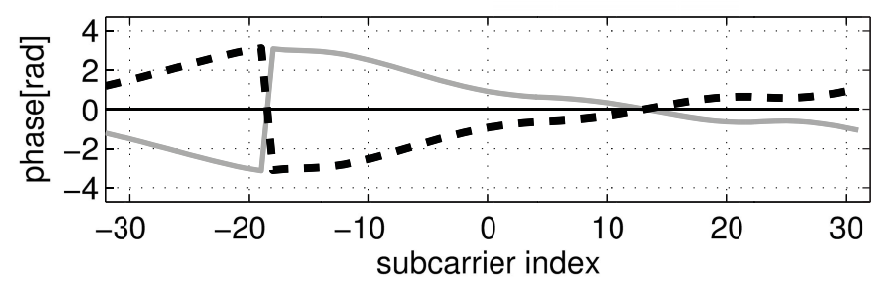

(c)

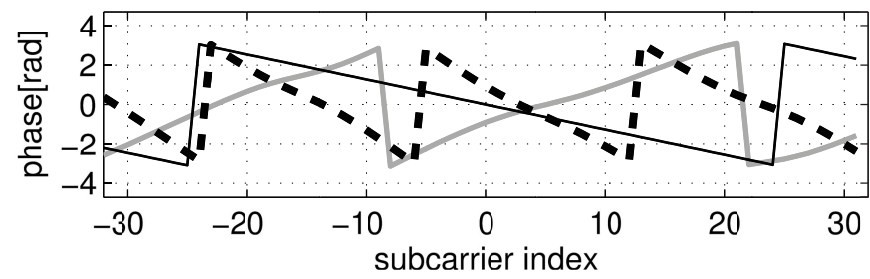

Fig. 2. (a) Frequency response of the channel (gray solid line), conjugate of the channel (black dashed line), and TR equivalent of the round-trip channel (black solid line). Magnitude spectra (a) and phase spectra without (b) and with (c) timing offsets $\zeta_{A}=-2.5$ and $\zeta_{B}=-1.2$ (hence $\delta=-1.3$ ). (b) In absence of timing offsets, the phase response of the TR equivalent of the round-trip channel is zero. (c) When timing offsets are present, a linear phase rotation appears that is proportional to the difference of the timing errors.

response of a multipath channel with the coefficient vector $\mathbf{h}=[1.75-\jmath 1.37,0.66+j 7.22,0.95-\jmath 1.02,-1.01+$ $J 0.89,1.47-j 0.78,]^{\mathrm{T}}$ its conjugate, and the TR equivalent channel after the round-trip flight of the signal. In the absence of timing errors, the TR equivalent frequency response of the channel corresponding to the round-trip flight of the signal is of zero phase [see Fig. 2(b)]. If symbol-timing errors are present, after round-trip flight of the signal, a linear phase rotation across OFDM subcarriers appears [see Fig. 2(c)]. The phase rotation is proportional to the difference of the symbol timing errors at the two wireless terminals $\delta=\left(\zeta_{A}-\zeta_{B}\right)$ that appears in (5), and thus its effect can be easily eliminated. Without TR, the channel phase would impair the linear phase rotation caused by the symbol-timing errors at the two radios, thus making its estimation extremely difficult. TR technique yields a linear phase of the two-way channel, regardless of the channel being minimum phase, maximum phase, or mixed phase. Thus, it can operate reliably in demanding multipath propagation environments such as indoors.

The TR technique is described by expressing the signal in each stage of the TS procedure.

1) Radio A transmits an OFDM signal, containing the frequency-domain data points $\left\{\mathcal{X}_{n}\right\}$.

2) Radio $B$ receives the signal with a timing error of $\zeta_{B}$ that produces a linear phase rotation across OFDM subcarriers. The received data point on the $n$th subcarrier 
at Radio B is written as

$$
\mathcal{Y}_{n}=\mathcal{H}_{n} \mathcal{X}_{n} \exp \left(J \frac{2 \pi n}{N} \zeta_{B}\right)+\mathcal{W}_{n}, n \in \mathbb{A}
$$

3) The received data points at Radio B are complex conjugated and transmitted back to Radio A. Complex conjugation in frequency domain is equivalent to TR and complex conjugation in time domain. The complex conjugated data point on the $n$th subcarrier is written as

$$
\mathcal{Y}_{n}^{*}=\mathcal{H}_{n}^{*} \mathcal{X}_{n}^{*} \exp \left(-j \frac{2 \pi n}{N} \zeta_{B}\right)+\mathcal{W}_{n}^{*}
$$

4) At Radio A, the OFDM signal is received with a symboltiming synchronization error of $\zeta_{A}$. The received data point is given by

$$
\begin{aligned}
\mathcal{Z}_{n}= & \mathcal{H}_{n} \mathcal{Y}_{n}^{*} \exp \left(j \frac{2 \pi n}{N} \zeta_{A}\right)+\mathcal{V}_{n} \\
= & \left|\mathcal{H}_{n}\right|^{2} \mathcal{X}_{n}^{*} \exp \left(j \frac{2 \pi n}{N}\left(\zeta_{A}-\zeta_{B}\right)\right) \\
& +\mathcal{H}_{n} \mathcal{W}_{n}^{*} \exp \left(j \frac{2 \pi n}{N} \zeta_{A}\right)+\mathcal{V}_{n}
\end{aligned}
$$

where $\mathcal{V}_{n}$ is the additive white Gaussian noise on the $n$th subcarrier that is added to the signal after the return trip.

5) Multiplying the received data point $\mathcal{Z}_{n}$ at Radio A by the corresponding transmitted data point $\mathcal{X}_{n}$ yields

$$
\begin{aligned}
\mathcal{Q}_{n}= & \mathcal{Z}_{n} \mathcal{X}_{n} \\
= & \left|\mathcal{H}_{n}\right|^{2}\left|\mathcal{X}_{n}\right|^{2} \exp \left(j \frac{2 \pi n}{N} \delta\right) \\
& +\mathcal{H}_{n} \mathcal{X}_{n} \exp \left(j \frac{2 \pi n}{N} \zeta_{A}\right) \mathcal{W}_{n}^{*}+\mathcal{X}_{n} \mathcal{V}_{n}
\end{aligned}
$$

It may be noticed in (9) that the phase of $\mathcal{Q}_{n}$ contains the parameter $\delta$ we are interested in [see (5)]. Note that the phase estimation in this paper is different from carrier phase estimation in OFDM systems presented, for example, in [49]. Carrier phase offset causes a constant phase shift over all OFDM subcarriers, whereas the symbol-timing error results in a linear phase rotation across the subcarriers. In this paper, we are interested in the estimation of the slope $\delta$ of this linear phase. Next section introduces a method to estimate the parameter $\delta$.

\section{Estimation of the Linear Phase SLOPE AND CRAMÉR-RAO BOUND}

In this section, the estimation of the phase slope parameter $\delta=\left(\zeta_{A}-\zeta_{B}\right)$ arising from the TR technique is addressed. First, the ML estimator of $\delta$ is derived. Then, a computationally efficient approach called FFT-WLS that is suitable for practical implementation is proposed. The CRLB is established to evaluate the performance of the proposed FFT-WLS algorithm.

\section{A. ML Estimation}

We consider the observation vector

$$
\mathbf{q}=\left[\mathcal{Q}-N_{\mathrm{a}}, \ldots, \mathcal{Q}_{N_{\mathrm{a}}}\right]^{\mathrm{T}}
$$

whose elements are given in (9). For the sake of simplicity, we assume that the transmitted data points are chosen from a constant modulus constellation, such as M-PSK. ${ }^{3}$ Therefore, $\left|\mathcal{X}_{n}\right|=1$, and (9) may be rewritten as

$$
\mathcal{Q}_{n}=\left|\mathcal{H}_{n}\right|^{2} \exp \left(J \frac{2 \pi n}{N} \delta\right)+\mathcal{U}_{n}, \quad n \in \mathbb{A}
$$

where $\mathcal{U}_{n}$ is the noise on the $n$th subcarrier, and is comprised of two noise terms as

$$
\mathcal{U}_{n}=\mathcal{H}_{n} \mathcal{X}_{n} \exp \left(J \frac{2 \pi n}{N} \zeta_{A}\right) \mathcal{W}_{n}^{*}+\mathcal{X}_{n} \mathcal{V}_{n}
$$

The noise samples $\mathcal{W}_{n}$ and $\mathcal{V}_{n}$ are white, mutually independent sequences, and they obey a zero-mean complex circular Gaussian distribution with variance of $\sigma^{2}$, i.e., $\mathcal{W}_{n}, \mathcal{V}_{n} \sim$ $\mathcal{C N}\left(0, \sigma^{2}\right)$. Therefore, from (12), we infer that the global noise on the $n$th subcarrier obeys $\mathcal{U}_{n} \sim \mathcal{C N}\left(0, \sigma_{n}^{2}\right)$, where $\sigma_{n}^{2}=$ $\sigma^{2}\left(1+\left|\mathcal{H}_{n}\right|^{2}\right)$. We assume that the frequency-domain channel $\left\{\mathcal{H}_{n}\right\}$, and timing errors $\zeta_{A}$ and $\zeta_{B}$ are deterministic. Hence, the unknown parameter of interest $\delta$ is also deterministic. The probability density function of the observation vector $\mathbf{q}$ conditioned on the parameter $\delta$ is

$$
\begin{aligned}
p(\mathbf{q} \mid \delta)= & \frac{1}{\pi^{2 N_{\mathrm{a}}}\left[\sigma^{2}\left(1+\left|\mathcal{H}_{n}\right|^{2}\right)\right]^{N_{\mathrm{a}}}} \\
& \times \exp \left(-\sum_{n \in \mathbb{A}} \frac{\left.\left.\left|\mathcal{Q}_{n}-\right| \mathcal{H}_{n}\right|^{2} e^{j \frac{2 \pi n}{N} \delta}\right|^{2}}{\sigma^{2}\left(1+\left|\mathcal{H}_{n}\right|^{2}\right)}\right) .
\end{aligned}
$$

Therefore, for $K$ i.i.d. observations, the log-likelihood function is written as (terms that do not depend on $\delta$ are ignored)

$$
\mathcal{L}(\delta)=-\operatorname{trace}\left\{\left(\mathbf{Q}-\mathbf{U}_{\delta} \mathbf{C}\right)^{\mathrm{H}} \Sigma_{\mathcal{U}}^{-1}\left(\mathbf{Q}-\mathbf{U}_{\delta} \mathbf{C}\right)\right\}
$$

where $\mathbf{Q}_{N \times K}=\left[\mathbf{q}_{0}, \ldots, \mathbf{q}_{K-1}\right]$, and the $k$ th observation vector $\mathbf{q}_{k}$ is as in (10). Matrix $\mathbf{C}$ is a $2 N_{\mathrm{a}} \times K$ matrix whose columns are equal to $\mathbf{a}=\left[\left|\mathcal{H}_{-N_{\mathrm{a}}}\right|^{2}, \ldots,\left|\mathcal{H}_{N_{\mathrm{a}}}\right|^{2}\right]^{\mathrm{T}}$. Matrix $\Sigma_{\mathcal{U}}$ is the $2 N_{\mathrm{a}} \times 2 N_{\mathrm{a}}$ diagonal covariance matrix of the global noise, whose diagonal elements are $\sigma_{n}^{2}, n \in \mathbb{A}$. Finally, matrix $\mathbf{U}_{\delta}$ is an $N \times N$ diagonal matrix with the elements $\left[\exp \left(-\jmath(2 \pi \delta / N) N_{\mathrm{a}}\right), \ldots, \exp \left(\jmath(2 \pi \delta / N) N_{\mathrm{a}}\right)\right]$ on its diagonal. The ML estimate of $\delta$ is obtained as

$$
\hat{\delta}_{\mathrm{ML}}=\arg \max _{\delta \in[-L, L]} \mathcal{L}(\delta) .
$$

The function $\mathcal{L}(\delta)$ (14) exhibits several local maxima. Its global maximum may be found by performing a grid search with sufficient resolution in the interval $[-L, L]$.

\section{B. Practical Algorithm for Linear Phase Estimation}

In this subsection, a computationally attractive algorithm for accurately estimating the linear phase slope $\delta$ is proposed. The ML algorithm in (15) requires an exhaustive search over the parameter of interest $\delta$, and therefore is not suitable for practical implementation. The search may be avoided by exploiting the structure of the observation vector $\mathbf{q}$ (10). The

\footnotetext{
${ }^{3}$ This is not a limitation of our algorithm. For QAM constellations, the derivation is very similar, but a multiplicative constant corresponding to the squared magnitude of the constellation point needs to be included.
} 
delay to be estimated is decomposed into an integer part and a fractional part

$$
\delta=\delta_{\text {int }}+\delta_{\text {frac }}, \quad \delta_{\text {int }} \in \mathbb{Z}, \quad\left|\delta_{\text {frac }}\right|<1 .
$$

First, the integer part $\delta_{\text {int }}$ is estimated by using FFT, and then, the remaining fractional delay $\delta_{\text {frac }}$ is estimated by using a WLS approach. For brevity, we call the proposed algorithm FFT-WLS.

The algorithm consists of the following steps.

1) First, each element $\mathcal{Q}_{n}$ (11) of the observation vector q (10) is power-normalized, i.e., the channel amplitude information is discarded. The amplitude information will be considered later in the algorithm derivation. Then, a phase-centering operation is performed, such that the middle subcarrier has zero phase. The phase slope $\delta$ that we are interested in remains unchanged after these first two operations.

2) The resulting vector $\overline{\mathbf{q}}(\delta)$ exhibits a Vandermonde structure similar to the columns of the FFT matrix, and noise

$$
\overline{\mathbf{q}}(\delta)=\left[\exp \left(-J \frac{2 \pi \delta}{N} N_{\mathrm{a}}\right), \ldots, \exp \left(J \frac{2 \pi \delta}{N} N_{\mathrm{a}}\right)\right]^{\mathrm{T}}+\overline{\mathbf{u}}
$$

where $\overline{\mathbf{u}}$ is a white Gaussian noise sequence whose components are $\overline{\mathcal{U}}_{n} \sim \mathcal{C N}\left(0, \sigma_{n}^{2} /\left|\mathcal{Q}_{n}\right|^{2}\right), n \in \mathbb{A}$.

3) Although the phase is linear across the spectrum, it cannot be estimated directly using a line fitting technique, due the wrapping that occurs between $-\pi$ and $\pi$, as seen in Fig. 2(c). To avoid this problem, FFT operation may be used. The phase slope $\delta$ is estimated with a resolution limited to integers by detecting the corresponding peak in the FFT spectrum of $\overline{\mathbf{q}}(\delta)$ (similar to beamforming). The resolution may be increased beyond integer values by taking a larger size FFT, but in general that is undesirable. To be able to use the $N$-point FFT operation that is already implemented in the OFDM receiver, the remaining fractional part of the delay needs to be estimated separately.

4) The contribution of the estimated integer delay $\hat{\delta}_{\text {int }}$ is removed from the vector $\overline{\mathbf{q}}(\delta)$ as follows:

$$
\overline{\mathbf{g}}\left(\delta-\hat{\delta}_{\text {int }}\right)=\overline{\mathbf{q}}(\delta) \odot \overline{\mathbf{q}}^{*}\left(\hat{\delta}_{\text {int }}\right)
$$

where $\overline{\mathbf{q}}\left(\hat{\delta}_{\text {int }}\right)$ has the same structure as $\overline{\mathbf{q}}(\delta)$, except for the fact that $\hat{\delta}_{\text {int }}$ is used in place of $\delta$, and $\odot$ denotes the Schur-Hadamard product. Consequently, the remaining timing error is fractional, i.e., $\left|\delta-\hat{\delta}_{\text {int }}\right| \leq 1$, and no phase-wrapping problem occurs. Hence, it may be estimated by using WLS.

5) The phase angles corresponding to $\overline{\mathbf{g}}\left(\delta-\hat{\delta}_{\text {int }}\right)$ have the form

$$
r_{n}=\angle g_{n}=\frac{2 \pi}{N} n\left(\delta-\hat{\delta}_{\text {int }}\right)+\eta_{n}, \quad n \in \mathbb{A}
$$

where $\eta_{n}$ is the phase deviation due to the noise on the $n$th subcarrier.

6) To increase the robustness to noise, the fitting errors may be weighted according the SNR on each subcarrier.
The WLS estimate of fractional part of the delay is obtained as

$$
\hat{\delta}_{\text {frac }}=\frac{N}{2 \pi}\left(\mathbf{n}_{\mathrm{a}}^{\mathrm{T}} \mathbf{W} \mathbf{n}_{\mathrm{a}}\right)^{-1} \mathbf{n}_{\mathrm{a}}^{\mathrm{T}} \mathbf{W r}
$$

where the vector $\mathbf{n}_{\mathrm{a}}=\left[-N_{\mathrm{a}}, \ldots, N_{\mathrm{a}}\right]^{\mathrm{T}}$ contains the indices of the active subcarriers, and the vector $\mathbf{r}=$ $\left[r_{-N a}, \ldots, r_{N a}\right]^{\mathrm{T}}$ contains the phase angles in (19). The weighting matrix $\mathbf{W}$ is diagonal, with the diagonal elements $\left|\hat{H}_{n}\right|^{4} /\left(1+\left|\hat{H}_{n}\right|^{2}\right)$, where $\left|\hat{H}_{n}\right|^{2}$ represents the estimated squared channel frequency response at the $n$th subcarrier, $n \in \mathbb{A}$. These weights were derived from the ML estimator (14) taking into account the normalization done in step 1). The same weights appear also in the expression of the Fisher information, as it will be seen in the next Section IV-C. The final FFT-WLS estimate of the delay is obtained as $\hat{\delta}=\hat{\delta}_{\text {int }}+\hat{\delta}_{\text {frac }}$.

When the number of available observations $K>1$, their average is used. Therefore, the corresponding SNR increase is equal to $10 \log K \mathrm{~dB}$. The complexity of the proposed FFT-WLS algorithm is at most of order $\mathcal{O}(N L+K)$. The computational complexity of the ML algorithm in Section IV-A is much higher, at least of order $\mathcal{O}\left(K N^{2} L\right)$. The strength of the proposed FFT-WLS algorithm is not just a reduced number of operations, but the fact that allows for highly parallel computation, unlike the ML estimator. In addition, we show in simulations that the proposed FFT-WLS algorithm is more robust to large carrier frequency offsets than the ML algorithm in Section IV-A.

\section{Cramér-Rao Lower Bound}

CRLB is derived to evaluate the MSE performance of the proposed FFT-WLS algorithm used to estimate the slope $\delta$ of the linear phase resulting from the TR technique. Since the $\mathrm{A} / \mathrm{D}$ - and D/A-converters in a transceiver use the same clock as the system clock, the clock values precisely match the samples at the transmission/reception time instant. Therefore, any error in the estimation of the phase parameter $\delta=\zeta_{A}-\zeta_{B}$ results directly into an error in the estimation the clock-offset $\Delta \tau$ (5). Consequently, CRLB may be used to evaluate the performance of the developed TS method. From (14), the Fisher information is

$$
\mathcal{I}(\delta)=2\left(\frac{2 \pi}{N}\right)^{2} \frac{K}{\sigma^{2}} \sum_{n \in \mathbb{A}} \frac{\left|\mathcal{H}_{n}\right|^{4}}{1+\left|\mathcal{H}_{n}\right|^{2}} n^{2} .
$$

Therefore, $\operatorname{Var}(\hat{\delta}) \geq 1 / \mathcal{I}(\delta)$. From (5), variance of $\widehat{\Delta \tau}$ can be written in terms of the variance of $\hat{\delta}$ as $\operatorname{Var}(\widehat{\Delta \tau})=$ $\left(T_{s}^{2} / 4\right) \operatorname{Var}(\hat{\delta})$. Therefore, we have

$$
\operatorname{Var}(\widehat{\Delta \tau}) \geq \frac{T_{s}^{2}}{4} \frac{1}{\mathcal{I}(\delta)} .
$$

\section{OFDM-RANGING USING TR-TS METHOD}

Ranging based on time measurements is very challenging due to the fact that timing errors are multiplied by the propagation speed, thus resulting into very large distance errors. In this section, we aim at estimating the distance between Radios $\mathrm{A}$ and $\mathrm{B}$ using the TR time-synchronization (TR-TS) method 
proposed in Section III. The setup is the same as in Fig. 1. From (4), the distance between the two radios is obtained as

$$
\hat{d}_{A B}=v\left[\left(c_{A}\left(t_{3}\right)-\hat{\zeta_{A}} T_{s}\right)-\left(c_{B}\left(t_{2}\right)-\widehat{\Delta \tau}\right)\right] .
$$

The timing offset $\Delta \tau$ is estimated using (5). The OFDM symbol-timing synchronization error at Radio A, $\zeta_{A}$ is unknown, and needs to be estimated as well. It corresponds to the difference detected between the beginning of the OFDM symbol and the true ToA of the line-of-sight path. In this paper, matrix mencil method is employed for the high-resolution multipath delay estimation [15]-[17], [42]. The main motivation for using the matrix pencil method is that it possesses superior time resolution compared with the classical timing estimation methods (e.g., based on FFT or correlation [32], [33], [50]) that are limited to the sampling period resolution. In addition, it uses the discrete channel frequency response, which makes it very suitable for multicarrier modulations such as OFDM.

\section{Practical Considerations}

In this section, practical aspects related to the proposed TS and ranging methods are addressed and the underlying assumptions of the proposed methods are justified. Subsection VI-A deals with the various delays in message delivery between two radios. Subsection VI-B provides arguments for the channel reciprocity assumption required in the proposed TR technique. A simple method to deal with the clock drifts is given in Section VI-C. The impact of the carrier frequency offset on the proposed methods is analyzed in Section VI-D.

\section{A. Time-Stamps and Sources of TS Error}

The most significant source of error in TS algorithms stems from the nondeterminism in message delivery latency time. By appropriate time-stamping, however, this delay can be considered deterministic and be very accurately compensated for. The message delay may be decomposed into distinct contributing factors [5], [19], [25].

1) Send Time - the time spent at the transmitter to construct a packet at the application layer and to pass it to the lower layers for transmission. That is, the delay experienced by the packet to reach the MAC layer from the application layer.

2) Access Time - the time spent waiting at the MAC layer for the packet to access the channel. The delay, which is specific to wireless networks, results from the property of the common medium for packet transmission. The contribution of this factor to the message delay is very significant and it is highly variable in nature.

3) Transmission Time - the time during which the OFDM packet is transmitted symbol by symbol at the physical layer over the wireless link. This delay is deterministic in nature and can be estimated using the packet size and the processing speed of the radio [19].

4) Propagation Time - the time spent by the packet to travel from the sender to the receiver over the wireless channel. In most of the clock-synchronization literature, this time is usually considered negligible. However, propagation time is the crucial information in ranging applications and needs to be estimated accurately.

5) Reception Time - the time taken in receiving the bits and passing them to the MAC layer. Like the transmission time, the reception time is deterministic in nature and can be accurately estimated [19].

6) Receive Time - the time spent for processing at the receiver's network interface. It represents the time taken from the actual reception of the packet and actually informing the application layer of the arrival of the message.

Among the delays mentioned above, Send Time, Access Time, Propagation Time, and Receive Time are random delays, whereas Transmission Time and Reception Time are deterministic delays.

In this paper, the effects of random delays are mitigated by time-stamping the OFDM packets at the MAC layer [19], instead of the application layer. This is because the delay experienced by the packet to arrive at the MAC layer from the application layer is highly variable in nature due to the software delays introduced by the underlying operating system. Hence, the time spent at the transmitters before packet transmission becomes equal to Transmission Time instead of the total of (Send + Access + Transmission Time). Similarly, by time-stamping the packet at the MAC layer at the receivers, Receive Time is removed, and only Reception Time remains. Since Transmission Time and Reception Time are deterministic delays, they can be used in the proposed TS and ranging methods. This fact was also confirmed by experiments using a prototype wireless platform.

\section{B. Channel Reciprocity}

The proposed TR-TS technique relies on the reciprocity property of the channel at the base-band input and output of the communication system [9]. This property arises from the fact that the electromagnetic waves traveling in both directions in the channel will experience the same perturbations, such as reflection, refraction, and diffraction. In other words, the impulse response of the channel between two radios operating on the same frequency spectrum should be the same regardless of the direction of the transmission [51]. In this section, some key factors undermining the validity of the channel reciprocity assumption are examined.

In general, there are two main detrimental factors causing channel nonreciprocity. One of them is the Doppler spread that is attributed to a channel with short coherence time caused by mobility of radios and scatterers. The TS and ranging methods in this paper are applicable in scenarios where the distances and the velocities are low, such as indoors, but they may extend to other scenarios as well. This is because in most existing terrestrial wireless systems, the channel coherence time is considerably longer than the signal roundtrip time-of-flight. Therefore, the channel may be considered quasi-stationary over the observation period, and the channel reciprocity assumption holds. Two different practical scenarios are considered below. 
1) WLAN Scenario: Consider two radios located at a distance of $30 \mathrm{~m}$ from each other. Suppose that each OFDM packet, used for ranging, contains 20 OFDM symbols plus the preamble, and the base-band sampling frequency is $20 \mathrm{MHz}$ (based on the WLAN standard). The digital processing delay at the second radio is slightly higher than the time required to buffer the entire OFDM packet, since the processing usually starts as soon as there is enough data in the buffer. Therefore, the digital processing delay is about $100 \mu$ s or slightly higher. There are also delays caused by analog filters, which amount to no more than a couple of $\mu$ s. Additionally, there is a delay due to the switch of the transceiver from the receiver $(\mathrm{Rx})$ to transmitter (Tx) mode. The Rx-Tx turnaround time with a chip is typically around $7 \mu \mathrm{s}$. This time may change between less than $5 \mu$ s to no more than $20 \mu$ s for WLAN standard [52]. Hence, the total processing delay amounts to approximately $120 \mu \mathrm{s}$. The propagation delay for a distance of $30 \mathrm{~m}$ is about $100 \mathrm{~ns}$. Consequently, the round-trip time-of-flight of the signal from one radio to the other radio will be around $120 \mu$ s. Now, if the maximum velocity in the indoor environment is equal to, let us say $36 \mathrm{~km} / \mathrm{h}$ in an extreme case, the Doppler frequency shift will be around $80 \mathrm{~Hz}$, for a carrier frequency of $2.45 \mathrm{GHz}$. That corresponds to a coherence time of $5.3 \mathrm{~ms}$ [53], which is 45 times longer than the round-trip time-of-flight of the OFDM packet. Therefore, the Doppler shift is not a critical factor for the proposed algorithm in indoor scenarios. This was also confirmed by the real-world experiments included in this paper.

2) Vehicular Ad Hoc Network Scenario: Consider the $5.9 \mathrm{GHz}$ IEEE $802.11 \mathrm{p}$ standard for vehicular communications. Let intervehicle distance be $10 \mathrm{~km}$ (in practice, the radio range in urban areas is of order few hundreds of meters). The corresponding round-trip time-of-flight of $66.6 \mu \mathrm{s}$, to which the processing time of around $120 \mu \mathrm{s}$ is added, whereas the channel coherence time at a speed of $300 \mathrm{~km} / \mathrm{h}$ is $258.2 \mu \mathrm{s}$. Therefore, the channel stays reciprocal during the signal round-trip.

The second factor impairing the reciprocity of the channel is the nonidealities at the transmitter and receiver front-ends. In general, RF front-ends of the wireless devices have very similar frequency-domain characteristics. This is due to the fact that they all target a box-shaped magnitude spectrum of the $\mathrm{Tx} / \mathrm{Rx}$ filters (constant gain) and a linear phase across all the useful frequency band. The phase slope is related to the group delay of the filters, and can be removed in a calibration process. Thus, the influence of the front-end hardware becomes negligible. Sampling clock deviation may also impact negatively the reciprocity property of the wireless channel. This deviation is very small in hardware nowadays, because over-sampling is always used to ease the requirements for the digital filters in the cutoff region. For example, in the case of WLAN base-band signal whose normal sampling rate is $20 \mathrm{MHz}$, assuming an over-sampling factor of 4 , one sample deviation corresponds to around $1.9 \mathrm{~m}$. The deviation of one sample is an upper bound, and it is caused by random jitter in the clock reading. In average, the corresponding error is smaller. Therefore, it is reasonable to assume that the wireless channel is reciprocal [9] for low-mobility ranging systems.

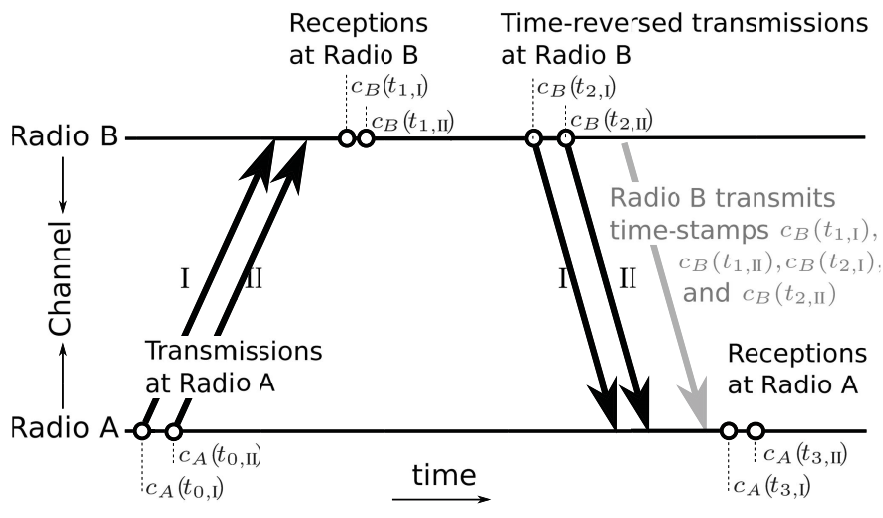

Fig. 3. Setup of the clock drift compensation procedure. Two messages named I and II are transmitted from Radio A to Radio B. The corresponding receive time-stamps $c_{B}\left(t_{1, \mathrm{I}}\right), c_{B}\left(t_{1, \mathrm{II}}\right)$, and transmit time-stamps $c_{B}\left(t_{2, \mathrm{I}}\right), c_{B}\left(t_{2, \mathrm{II}}\right)$, respectively, are transmitted back to Radio A along with the corresponding time-reversed signals.

In Section VII, we show real-world measurements validating this assumption.

\section{Eliminating the Effect of Clock Drifts}

In practice, the quartz crystals in each of the machines may run at slightly different frequencies, due to draining batteries, temperature variations, etc. This causes the clock values at the two radios to gradually diverge from each other over time. The clocks drifts take place very slowly. Their impact on the proposed algorithm during a such a short time as a single round-trip time-of-flight can be considered to be negligible. However, we present a simple method that corrects these small drifts. In our proposed TS method, the error caused by the clock drift between the two radios will appear in the processing delay at Radio B, thus, making the estimate of the clock-offset erroneous. The processing delay measured at Radio B corresponds to a different delay with respect to the clock of Radio A (see Fig. 1), i.e., $\left[c_{A}\left(t_{2}\right)-c_{A}\left(t_{1}\right)\right]=$ $\gamma\left[c_{B}\left(t_{2}\right)-c_{B}\left(t_{1}\right)\right]$, where $\gamma$ is the scale parameter that shows the relative drift of the two clocks (ideally, $\gamma=1$ ). To correct the clock drift errors in the proposed TR TS method, we employ a method stemming from the DEVAC method in [40]. However, it is extended by employing the TR technique to improve the clock drift estimation accuracy. Two consecutive messages, Messages I and II are transmitted from Radio A to Radio $\mathrm{B}$ at time instants $t_{0, \mathrm{I}}$ and $t_{0, \mathrm{II}}$, respectively (see Fig. 3). At Radio B, the messages are received at time instants $t_{1, \mathrm{I}}$ and $t_{1, \mathrm{II}}$ with symbol-synchronization errors $\zeta_{B, \mathrm{I}}$ and $\zeta_{B, \mathrm{II}}$, respectively. Along with the time-stamps $c_{B}\left(t_{1, \mathrm{I}}\right)$ and $c_{B}\left(t_{1, \text { II }}\right)$, Radio B also transmits the two time-reversed version of the OFDM symbols. Incorporating the scale parameter $\gamma$ in the proposed TS algorithm, the clock-offset estimate is given by

$$
\begin{aligned}
& \widehat{\Delta \tau}=\frac{1}{2}( {\left[(2-\hat{\gamma}) c_{B}\left(t_{1, \mathrm{II}}\right)+\hat{\gamma} c_{B}\left(t_{2, \mathrm{II}}\right)\right] } \\
&\left.-\left[c_{A}\left(t_{3, \mathrm{II}}\right)+c_{A}\left(t_{0, \mathrm{II}}\right)\right]+\hat{\delta}_{\mathrm{II}} T_{s}\right)
\end{aligned}
$$


where $\hat{\gamma}$ is estimated as ${ }^{4}$

$$
\hat{\gamma}=\frac{c_{A}\left(t_{0, \mathrm{II}}\right)-c_{A}\left(t_{0, \mathrm{I}}\right)}{c_{B}\left(t_{1, \mathrm{II}}\right)-c_{B}\left(t_{1, \mathrm{I}}\right)} .
$$

The phase slope $\hat{\delta}_{\text {II }}$ corresponding to Message II is estimated using the FFT-WLS algorithm derived in Section IV-B.

\section{Impact of the Carrier Frequency Offset on the Proposed Timing Estimation Algorithm}

In this section, the impact of the carrier frequency offset (CFO) on the proposed timing estimation algorithm is studied. In indoor environments, the Doppler effect caused by the relative radial motion of the two radios has a negligible effect in terms of CFO [54, Ch 5]. This is also justified in Section VI-B. However, the frequency mismatch between the local oscillators used to perform the up-conversion/down-conversion at the two radios may generate significant CFO. In general, the OFDM receivers possess mechanisms for reducing the CFO such that the data symbols can be demodulated reliably. Typically, the residual frequency offset is required to be less than $10 \%$ of the intercarrier spacing [54, Ch 5]. Let $\epsilon$ be the normalized frequency offset, relative to the intercarrier spacing $1 /\left(N T_{s}\right)$, i.e., $\epsilon=\Delta f N T_{s}$, where $\Delta f$ is the frequency deviation measured in Hz. In this analysis, different residual CFOs $\epsilon_{A}$ and $\epsilon_{B}$ are assumed after the CFO compensation at the two radios, respectively.

In presence of the CFOs $\epsilon_{A}, \epsilon_{B}$ and the timing errors $\zeta_{A}, \zeta_{B}$ at the Radios $\mathrm{A}$ and $\mathrm{B}$, respectively, the sequence corresponding to the TR algorithm that contains the phase rotation $\delta=\zeta_{A}-\zeta_{B}$ in which we are interested in [see (9)] is given in $^{5}$

$$
\begin{aligned}
\tilde{\mathcal{Q}}_{n}= & \left|\mathcal{H}_{n}\right|^{2}\left|\mathcal{X}_{n}\right|^{2} \exp \left[J \frac{2 \pi n}{N}\left(\zeta_{A}-\zeta_{B}\right)\right] \frac{\sin \left(\pi \epsilon_{A}\right)}{N \sin \left(\pi \epsilon_{A} / N\right)} \\
& \times \frac{\sin \left(\pi \epsilon_{B}\right)}{N \sin \left(\pi \epsilon_{B} / N\right)} \exp \left(J \pi \frac{N-1}{N}\left(\epsilon_{B}-\epsilon_{A}\right)\right) \\
& +\mathcal{H}_{n}^{*}\left|\mathcal{X}_{n}\right|^{2} \sum_{\substack{l \neq n \\
l=-N_{\mathrm{a}}}}^{N_{\mathrm{a}}} \mathcal{H}_{l} \exp \left[J \frac{2 \pi}{N}\left(n \zeta_{A}-l \zeta_{B}\right)\right] \\
& \times \frac{\sin \left[\left(l-n-\epsilon_{A}\right) \pi\right]}{N \sin \left[\left(l-n-\epsilon_{A}\right) \pi / N\right]} \frac{\sin \left[\left(l-n+\epsilon_{B}\right) \pi\right]}{N \sin \left[\left(l-n+\epsilon_{B}\right) \pi / N\right]} \\
& \times \exp \left[J \pi \frac{N-1}{N}\left(2 l-2 n+\epsilon_{B}-\epsilon_{A}\right)\right] \\
& +\mathcal{H}_{n} \mathcal{X}_{n} \exp \left[J \frac{2 \pi n}{N}\left(\zeta_{A}-\zeta_{B}\right)\right] \frac{\sin \left(\pi \epsilon_{A}\right)}{N \sin \left(\pi \epsilon_{A} / N\right)} \\
& \times \sum_{l \neq n}^{N_{\mathrm{a}}} \mathcal{H}_{l}^{*} \mathcal{X}_{l}^{*} \frac{\sin \left[\left(l-n-\epsilon_{B}\right) \pi\right]}{N \sin \left[\left(l-n-\epsilon_{B}\right) \pi / N\right]} \\
& \times \exp \left[j \pi \frac{N-1}{N}\left(n-l+\epsilon_{\mathrm{a}}-\epsilon_{A}\right)\right]
\end{aligned}
$$

\footnotetext{
${ }^{4}$ The denominator in (25) should include an extra-term $\left(\zeta_{B, \mathrm{I}}-\zeta_{B, \mathrm{II}}\right) T_{s}$, i.e., the difference of timing errors at Radio B corresponding to the two Messages I and II, respectively. Due to the fact that in practice $\left(\zeta_{B, \mathrm{II}}-\zeta_{B, \mathrm{I}}\right) T_{S} \ll$ $\left[c_{B}\left(t_{1, \mathrm{II}}\right)-c_{B}\left(t_{1, \mathrm{I}}\right)\right]$, this extra-term may be neglected.

${ }^{5}$ Proof is available on request. The initial phases of the main carriers were considered to be zero in (26), as they produce no intercarrier interference.
}

$$
\begin{aligned}
& +\mathcal{X}_{n} \sum_{l=-N_{\mathrm{a}}}^{N_{\mathrm{a}}} \mathcal{H}_{l} \exp \left[J \frac{2 \pi}{N}\left(n \zeta_{A}-l \zeta_{B}\right)\right] \\
& \times \frac{\sin \left[\left(l-n-\epsilon_{A}\right) \pi\right]}{N \sin \left[\left(l-n-\epsilon_{A}\right) \frac{\pi}{N}\right]} \\
& \times \sum_{\substack{l \neq n \\
l=-N_{\mathrm{a}}}}^{N_{\mathrm{a}}} \mathcal{H}_{p}^{*} \mathcal{X}_{p}^{*} \frac{\sin \left[\left(p-l-\epsilon_{B}\right) \pi\right]}{N \sin \left[\left(p-l-\epsilon_{B}\right) \pi / N\right]} \\
& \times \exp \left[j \pi \frac{N-1}{N}\left(2 l-p-n+\epsilon_{B}-\epsilon_{A}\right)\right] \\
& +\mathcal{X}_{n}\left[\tilde{\mathcal{W}}_{n}^{*}+\tilde{\mathcal{V}}_{n}\right] \text {. }
\end{aligned}
$$

The noise terms $\tilde{\mathcal{V}}_{n}$ and $\tilde{\mathcal{W}}_{n}$ in (26) are given in Appendix. The first term in (26) shows the amplitude reduction and the phase rotation caused by the CFOs $\epsilon_{A}, \epsilon_{B}$ on the $n$th subcarrier, as well as the phase rotation caused by the timing errors $\zeta_{A}, \zeta_{B}$ at the two radios. This is the dominant term when $\epsilon_{A}, \epsilon_{B}$ are close to zero, and the SNR is high, as it will be seen in the simulations. For this term, the phase rotation caused by the CFOs is constant for all subcarriers, and therefore, it does not affect the slope of the phase caused by the timing errors. As the CFOs $\epsilon_{A}, \epsilon_{B}$ increase, the intercarrier interference becomes significant. The contribution of the adjacent tones to the $n$th subcarrier is shown by the second, third, and fourth terms. We show in simulations that the proposed TR technique performs very well for typical CFO values encountered in practice due to transceiver oscillator mismatches. The user mobility causes a minor Doppler frequency shift that does not impact the TR technique. For example, in the case of IEEE $802.11 \mathrm{a} / \mathrm{g}$ WLAN operating at $2.45 \mathrm{GHz}$, a speed of $36 \mathrm{~km} / \mathrm{h}$ causes a normalized CFO $\epsilon=2.6 \times 10^{-4}$. Another practical example is a $5.9 \mathrm{GHz}$ IEEE $802.11 \mathrm{p}$ vehicular network. Assuming that the relative speed of the two communicating vehicles is $300 \mathrm{~km} / \mathrm{h}$, the resulting normalized CFO is also negligible, $\epsilon=5.2 \times 10^{-3}$. When FFT-WLS is used for estimating the linear phase, at moderate SNR, CFO values up to 0.2 (20\% of the intercarrier spacing) can be tolerated. Therefore, the CFO does not cause any significant performance degradation to the proposed TR technique.

\section{Simulation Examples ANd Results Using Measured Channels}

To assess the performance of the proposed TS method, an IEEE 802.11a [52] WLAN communication system was considered. The OFDM signal consists of $N=64$ subcarriers out of which 52 are active (i.e., $N_{\mathrm{a}}=26$ ), and the cyclic prefix length is $L=16$. The base-band processing sampling rate is assumed to be $20 \mathrm{MHz}$, which is equivalent to a sampling period of $50 \mathrm{~ns}$. A wireless platform was used to examine the validity of the assumptions in Section VI. In addition, real measured channels [55] are used to asses the performance of the proposed ranging method.

\section{A. Channel Reciprocity}

Channel reciprocity is a fundamental requirement for the application of the TR technique in the TS algorithm. To investigate the validity of the channel reciprocity, experiments 
were conducted on the wireless platform at Nokia Research Center, Espoo, Finland. The center carrier frequency is in the $2.45 \mathrm{GHz}$ ISM band, and the bandwidth (including the guard bands) is equal to $20 \mathrm{MHz}$. The TR technique was implemented on hardware, and the measurement setup is the one in Fig. 1. The measurements were obtained in an office environment with distances ranging between 5 and $15 \mathrm{~m}$. The transmitted and received OFDM data packets at the two radios are stored in a memory, and later processed in a computer. For the detection of the beginning of the first OFDM symbol, the OFDM packet was correlated with the long preamble. The rest of the symbols are detected by incrementing the index of the beginning of the first OFDM data part with a step size of $M=80$, i.e., the length of a OFDM symbol including the cyclic prefix.

Fig. 4 shows the magnitude and phase responses of the channels measured in a real office environment on the third floor of a four-story building. Each row in the figure corresponds to a different measurement scenario with the test number at the bottom of the row. The magnitude responses of the measured channels are shown on the left column of Fig. 4. The gray solid line shows the response of the complex conjugate of the channel $\left(\mathcal{H}^{\mathrm{fwd}}(f)\right)^{*}$ on the forward link (because it is complex conjugated at Radio B in the TR-TS method), and the dashed line shows the response of the channel $\mathcal{H}^{\text {ret }}(f)$ on the return link. The black solid line shows the round-trip TR equivalent response of the channel $\mathcal{H}^{\mathrm{TR}}(f)$. The magnitude spectra of the forward and return channels are very similar, therefore the relation $\left|\mathcal{H}^{\mathrm{TR}}(f)\right|=\left|\mathcal{H}^{\mathrm{fwd}}(f)\right|^{2}=\left|\mathcal{H}^{\text {ret }}(f)\right|^{2}$ is satisfied with high fidelity in practice. The right column of Fig. 4 shows the phase responses of the complex conjugate of the forward channel and the return channel. It can be seen that the phase responses of the forward and return channels have similar overall shapes. These phase responses incorporate the linear phase caused by the timing error at the two radios. The sum of the phase responses of the complex conjugate of the forward channel and the return channel is a linear phase that is caused by the difference of the two symboltiming synchronization errors at the two radios. This linear phase corresponds to the round-trip TR equivalent channel and remains linear regardless of the shape of the phase response of the one-way channel, as explained in Section III. Tests 1-4 correspond to stationary Radios A and B, whereas tests 5-7 corresponds to stationary Radio A and moving Radio B. We may also notice that mobility does not have significant impact on the channel reciprocity property. The real measurements shown in Fig. 4 provide a strong practical argument for the validity of the reciprocity property of the channel in real-world systems and scenarios.

\section{B. Estimation of the Parameter $\delta$}

In this section, the estimation of the phase slope parameter $\delta$ arising from the TR technique is considered. The channel in Fig. 2 was used. The results have been computed by averaging over $M=10000$ independent realizations, and the number of observations is fixed to $K=1$, for each realization $m$. The employed quantitative performance measure is the
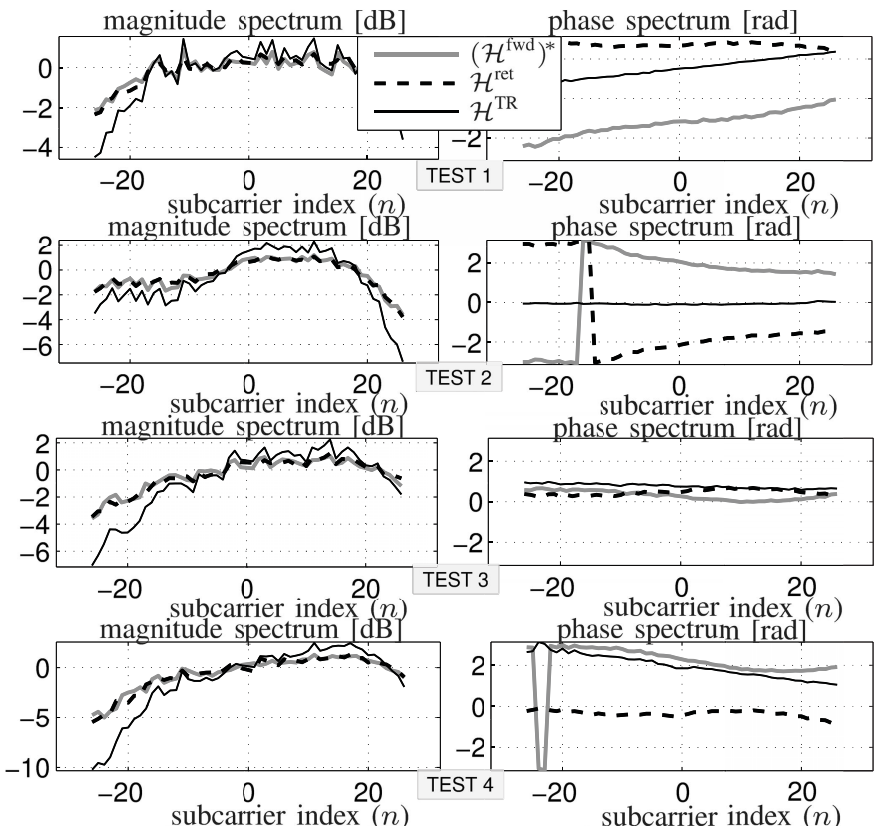

magnitude spectrum

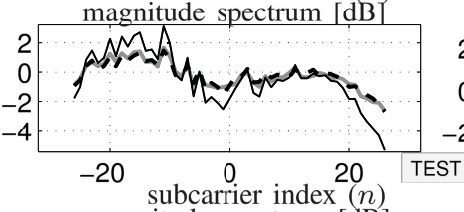

subcarrier index $(n)$
magnitude spectrum $[\mathrm{dB}]$

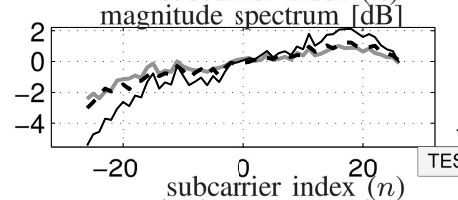

subcarrier index $(n)$
magnitude spectrum $[\mathrm{dB}]$

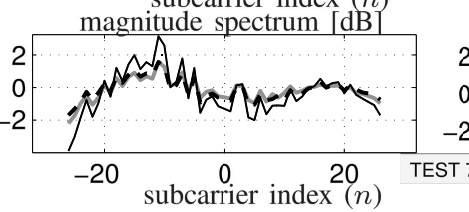

(a)
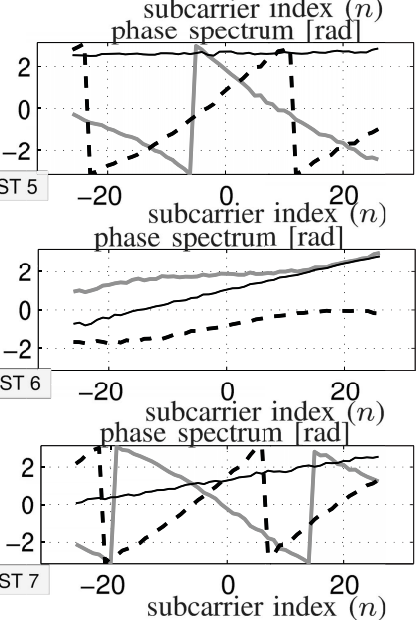

(b)

Fig. 4. Channel reciprocity plots with measured wireless channels. The gray solid line and black dashed line show the forward and return channels, respectively. The solid black line is shown the response of the TR equivalent channel. (a) Magnitude spectra of the forward and return channels are very similar. (b) Linear phase of the TR equivalent channel may be easily noticed.

root-mean-square error (RMSE) of the clock-offset estimates $\widehat{\Delta \tau}_{m}$

$$
\operatorname{RMSE}\{\widehat{\Delta \tau}\}=\sqrt{\frac{1}{M} \sum_{m=1}^{M}\left(\widehat{\Delta \tau}_{m}-\Delta \tau\right)^{2}}
$$

which is compared with the square-root of the CRLB (22). The results are shown in Fig. 5, for different SNR values. The solid gray line shows the square-root CRLB. The dashed line with circular markers shows the RMSE corresponding to the ML estimator in Section IV-A, in which the true channel is used for the estimation of $\delta$. The solid black line with triangular markers represents the RMSE corresponding to the FFT-WLS approach proposed in Section IV-B, in which the time-domain estimated channel is used. Fig 5 shows that the proposed FFTWLS and the ML estimator attain the CRLB at 6 and $4 \mathrm{~dB}$, respectively. 


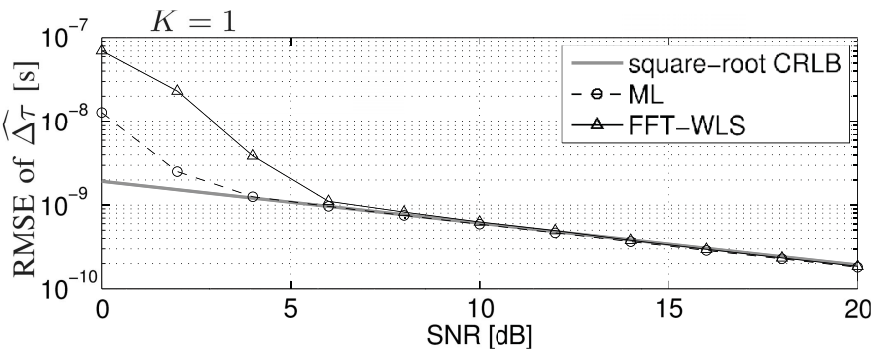

Fig. 5. Comparison between the clock-offset RMSE corresponding to the ML and FFT-WLS algorithms, respectively, and the square-root CRLB versus SNR, with $K=1$ observation. ML estimator attains the bound at approximately $4 \mathrm{~dB}$, whereas the proposed FFT-WLS algorithm, around $6 \mathrm{~dB}$.

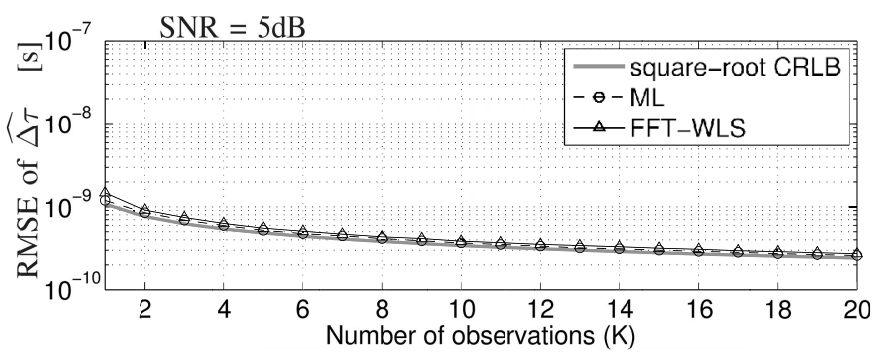

Fig. 6. Comparison between the clock-offset RMSE corresponding to the ML and FFT-WLS algorithms, respectively, and the square-root CRLB, versus sample size $K$, with fixed SNR $=5 \mathrm{~dB}$. The proposed FFT-WLS algorithm performs very close to the CRLB, even when single observation vector is used in the estimation.

Fig. 6 shows the RMSEs for different number of observations $K$ and a fixed signal-to-noise ratio of $5 \mathrm{~dB}$. Each OFDM symbol is considered as one observation. It can be seen that the RMSEs corresponding to both the proposed FFT-WLS algorithm and the ML estimator, respectively, are very close to the bound, even for a single observation vector.

\section{TR-TS}

In this section, we compare the proposed TR-TS method with three other methods, which are also based on the remote clock reading. The difference is in the synchronization method used to detect the reference for time-stamping the OFDM packet. In the first case, which can be considered as the direct implementation of the method in [19] for OFDM systems, the beginning of the data part of the first OFDM symbol is detected by correlating the cyclic prefix of the long preamble over the packet. In the TR-TS method, the same method is used for symbol-timing synchronization at the two radios. In the other two methods, the Schmidl-Cox [32] and Minn symbol-timing-synchronization [33] algorithms are employed to detect the beginning of the data part of the first OFDM symbol. The clock drifts are not relevant for this particular application because they take place very slowly, and the synchronization may be done more frequently.

Fig. 7 shows the RMSE of the clock-offset $\widehat{\Delta \tau}$ (27) for the aforementioned cases. The RMSE values are obtained using $M=10000$ random channels and for SNR values changing from 0 to $40 \mathrm{~dB}$. The dashed line with diamond markers shows the RMSE of $\widehat{\Delta \tau}$ for the symbol-timing synchronization

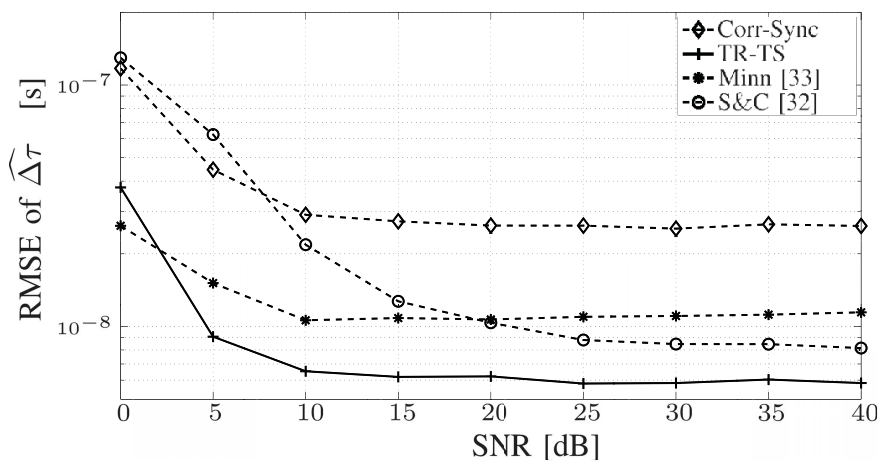

Fig. 7. RMSE of the clock-offset for different methods to detect the reference of time-stamping. The TR-TS method has a superior performance compared with other methods. S\&C in the legend refers to Schmidl-Cox algorithm [32].

based on the correlation of the cyclic prefix of the long preamble. The dashed lines with asterisk and circle markers show the RMSE of $\widehat{\Delta \tau}$ using Minn [33] and Schmidl-Cox [32] symbol-timing estimation algorithms, respectively. The solid line shows the RMSE of $\widehat{\Delta \tau}$ using the TR-TS method. The result shows the superiority of the TR-TS methods over the other three methods. It can also be seen that by employing the TR-TS method, without a complicated symbol-timing synchronization algorithm, a high-performance TS is achieved regardless of the multipath channel and even for low SNR values. As long as the channel stays reciprocal, the roundtrip equivalent channel exhibits a linear phase regardless of the multipath propagation. This allows for precise timing estimation in presence of multipath, unlike the correlationbased approaches that are limited to the sampling rate timing resolution, and cannot resolve closely spaced multipath components. Since the TR technique converts timing errors into a phase slope that can be estimated with high accuracy, the timing resolution goes beyond the Nyquist sampling period, without the need for oversampling.

\section{OFDM-Ranging Using the TR-TS Method}

In this section, we evaluate the performance of the proposed ranging method, first using simulations, and then using realworld measurements. To estimate the distance, the clock-offset $\Delta \tau$ and the timing error $\zeta_{A}$ are required in (23). The clockoffset is obtained from the proposed TR-TS method (5). The OFDM symbol timing error timing error $\delta_{A}$ is estimated with high resolution using a matrix pencil method [15]-[17], [42]. Note that the clock-offset estimation relies on the phase slope $\delta$, that is estimated using the proposed FFT-WLS algorithm in Section IV-B.

In the simulation, the clock-offset between the two radios is set to $\Delta \tau=115.6 \times T_{s}=5.78 \mu \mathrm{s}$, where $T_{s}=50 \mathrm{~ns}$. SNR is equal to $5 \mathrm{~dB}$ on both links. A randomly generated channel vector with the complex path gains $\mathbf{h}=[-0.6226+$ $\left.j 0.5322,0.5387+j 0.1356,0.1002-j^{0.1026}\right]^{\mathrm{T}}$, and path delays $\mathbf{t}=[0.0780,0.1693,0.1751]^{\mathrm{T}} \mu \mathrm{s}$ was used. Let the distance between the two radios be $d_{A B}=23.4 \mathrm{~m}$. After the round-trip of the signal, the estimated phase slope is $\hat{\delta}=-0.8215$ sampling periods. This yields a clock-offset estimate $\widehat{\Delta \tau}=5.7845 \mu \mathrm{s}$. The absolute error in the estimation 
of the clock-offset is, therefore, equal to $|\Delta \tau-\widehat{\Delta \tau}|=4.5 \mathrm{~ns}$, which is equivalent to a ranging error of $e_{d}=1.35 \mathrm{~m}$.

The impact of OFDM symbol timing errors on the ranging accuracy in this particular experiment is the following. Assuming perfect ToA estimation, the clock-offset estimation error due to solely ignoring $\delta$ would be $|\Delta \tau-\widehat{\Delta \tau}|=25 \mathrm{~ns}$, which results in a distance error of $e_{d}=7.5 \mathrm{~m}$. Therefore, to achieve the desired accuracy, it is necessary to take into account the parameter $\delta$ that corresponds to the difference of the time-stamping errors at the two radios. The OFDM symbol timing error estimated using the matrix pencil method was $\zeta_{A}=-1.4574$ sampling periods with respect to the beginning of the detected OFDM data part. Finally, using the estimated values of the clock-offset $\widehat{\Delta \tau}$, the phase slope $\hat{\delta}$, and the timing error $\zeta_{A}$, the estimated distance between the two radios is equal to $\hat{d}_{A B}=24.47 \mathrm{~m}$, i.e., the ranging error is reduced to $e_{d}=1.07 \mathrm{~m}$. The matrix pencil method is also subject to some error. Although the error is very small in this particular example, it happens to have a different sign compared with the error due to the clock-offset solely. Therefore, the result indicates that the ranging error is reduced compared with the case of perfect ToA estimation scenario. However, in practice, this is not always the case.

The impact of the ToA estimation error in this particular example is considerably higher. Without considering the OFDM symbol timing estimation error $\zeta_{A}$, the ranging error would be $e_{d}=21.86 \mathrm{~m}$.

The ranging based on the proposed TR-TS method has been further tested using channel measurements obtained during the measurement campaign within the WILATI project [55]. The measurement system is a dual-link MIMO channel sounder, which consists of one mobile transmitter and two receivers, namely Aalto receiver and Lund receiver. The center carrier frequency is equal to $5.3 \mathrm{GHz}{ }^{6}$ The channel transfer functions for the simulation are reconstructed using 64 active subcarriers with the corresponding bandwidth of $40 \mathrm{MHz}$. Single antenna element at both Tx and Rx was used.

Fig. 8 shows the floor plan of the atrium in the Computer Science building of Aalto University, Espoo, Finland, where part of the measurements were performed. The two receivers are marked by the black filled square and black circle markers in Fig. 8. The transmitter is moved over the routes marked by arrows number 5 and 2 . The transmitter and receivers are located at the same floor. In this section, the result of the ranging algorithm for the channels measured by the Lund receiver over route number 5 is presented. The estimated distances for subsequent consecutive measurement snapshots along the route are shown in Fig. 9 by gray solid line. The black dashed line shows the true distance between the transmitter and the receiver.

In Fig. 9(a), the TR-TS method is used for TS in the ranging procedure. In contrast, in Fig. 9(b), the TS is based on the remote clock reading and coarse synchronization that was initially performed by using the correlation method, that is, the mismatch of the reference points for time-stamping the OFDM packet at the two radios are not considered in the TS process.

\footnotetext{
${ }^{6}$ The $5 \mathrm{GHz}$ band is also standardized for IEEE $802.11 \mathrm{a} / \mathrm{h} / \mathrm{n}$ WLAN.
}

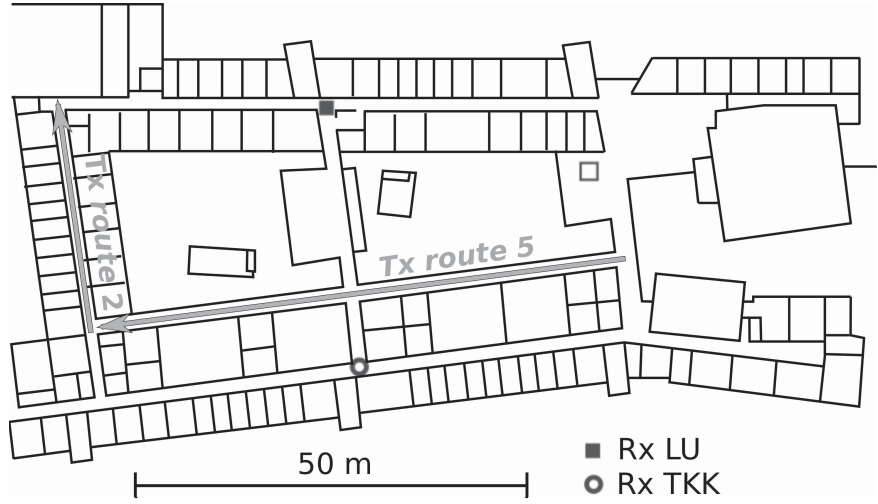

Fig. 8. Floor plan of the atrium scenario and measurement setup. The mobile transmitter moves along routes 5 and 2 shown by the gray arrows. The Lund receiver (LU Rx) and the Aalto receiver (TKK Rx) are marked with a filled black square and the black circle, respectively. The channel measurements along route number 5, and the LU Rx were used in this paper.
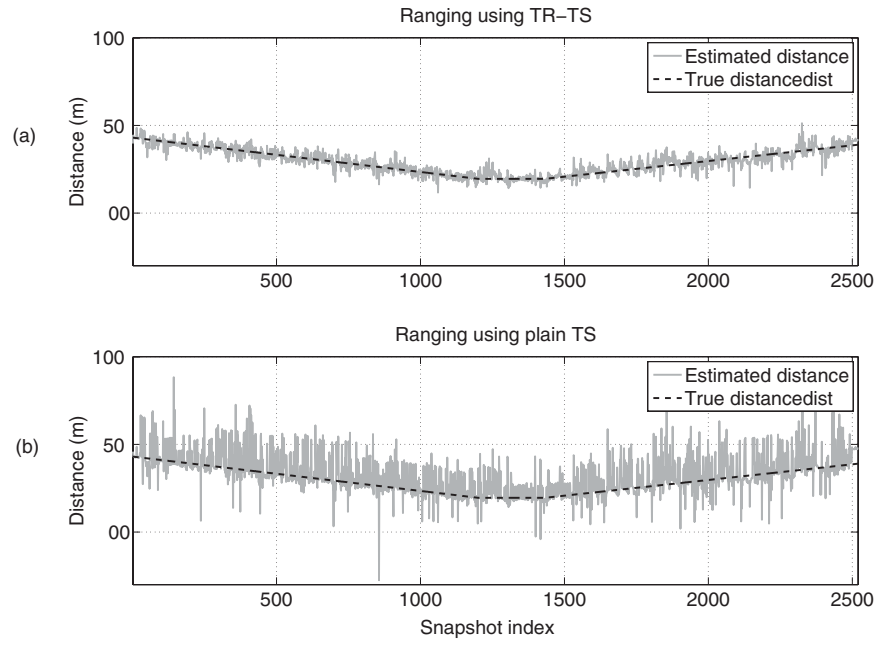

Fig. 9. Range estimation using real-world measurements. (a) Proposed TRTS method is used for TS and ranging. (b) TS based on the remote clock reading without considering the time-stamping errors. When using the TR-TS method, the effect of multipath is reduced, whereas in the other case, the distances are mostly overestimated.

In both cases, the matrix pencil method is used for the ToA estimation. In Fig. 9(b), it appears that the distances are mostly overestimated and the ranging algorithm yields range estimates with a bias of $3.5 \mathrm{~m}$, and a standard deviation of $8.5 \mathrm{~m}$. On the other hand, using the TR-TS method [Fig. 9(a)], the ranging errors are significantly smaller. The bias is $0.07 \mathrm{~m}$, and the standard deviation of the estimates of the distances is around $2.7 \mathrm{~m}$. The simulations show that the proposed TR-TS method provides significant performance gains in OFDM-based ranging systems employing high-resolution ToA estimation.

\section{E. Impact of the Carrier Frequency Offset}

The effect of the carrier frequency offsets at the two radios on the proposed algorithm is studied in terms of RMSE (27), for a fixed SNR value of $15 \mathrm{~dB}$. The results are averaged over $M=10000$ independent runs. In Fig. 10, different CFO values $\epsilon_{A}$ and $\epsilon_{B}$ are considered. There are four representative cases: the case when the two CFO have similar value with the same, or different sign, and also the cases when one of the 


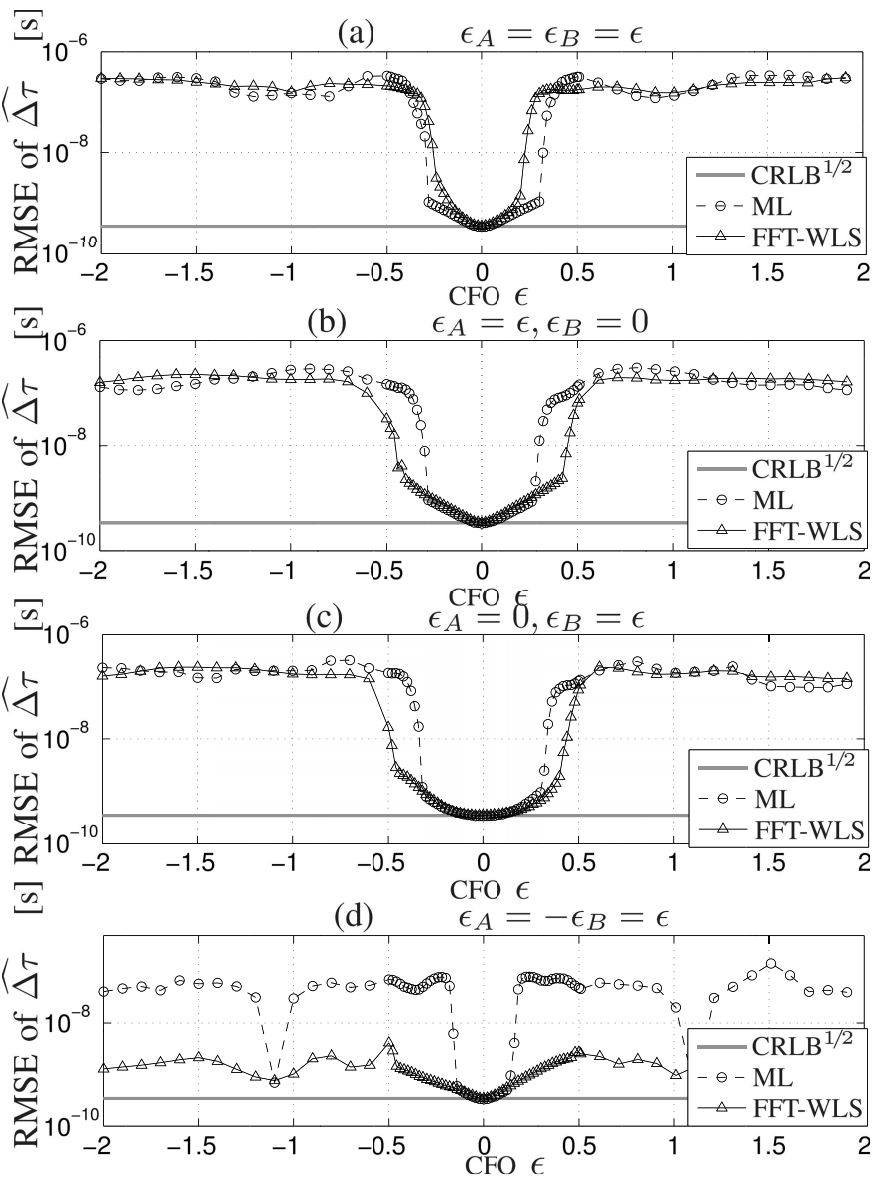

Fig. 10. Impact CFO on the clock-offset RMSE for the ML and FFT-WLS algorithms, respectively, versus the square-root CRLB, for different normalized CFO vales $\epsilon$. Four different cases are considered. (a) $\epsilon_{A}=\epsilon_{B}=\epsilon$, (b) $\epsilon_{A}=\epsilon, \epsilon_{B}=0$, (c) $\epsilon_{A}=0, \epsilon_{B}=\epsilon$, and (d) $\epsilon_{A}=-\epsilon_{B}=\epsilon$. FFT-WLS algorithm outperforms the ML algorithm in most of the cases.

CFOs dominates, and the other has a low value. These four cases may be general enough to capture the behavior of other combinations that include intermediate $\mathrm{CFO}$ values.

In the first case, both CFOs are equal, i.e., $\epsilon_{A}=\epsilon_{B}$, as shown in Fig. 10(a). The ML algorithm can tolerate residual CFO values up to $30 \%$ of the subcarrier spacing, while the FFT-WLS algorithm can tolerate normalized CFO values up to $20 \%$. Such values are usually too large in OFDM receivers after the CFO compensation operation because the symbol demodulation becomes impossible due the severe intercarrier interference. The case $\epsilon_{A}=\epsilon_{B}$ is the most undesirable case in terms of timing mean square error, but there is no particular reason why this should occur more often in practice.

When one of the residual CFOs (either $\epsilon_{A}$ or $\epsilon_{B}$ ) has small values (as often occurs in practice), the FFT-WLS algorithm outperforms the ML estimator, as shown in Fig. 10(b) and (c).

The case when the two CFOs have comparable values but opposite sign is the most frequent case in practice, due to the round-trip transmission, i.e., the $\mathrm{CFO}$ on the return channel has opposite direction. For this particular case, FFT-WLS outperforms ML estimator by far, and can tolerate very large CFO values, as shown in Fig. 10(d).

In the worst case (when $\epsilon_{A}=\epsilon_{B}$ ), FFT-WLS performs slightly worse than the ML. In all the other cases it outperforms the ML algorithm. In conclusion, the proposed FFT-WLS can handle practical CFO values. When the CFO values at the two radios do not exceed $20 \%$ of the intercarrier spacing, the first term in (26) that contains the useful linear phase dominates, and the phase slope can still be estimated with good accuracy.

\section{CONCLUSION}

In this paper, we introduce a two-way TS method for OFDM-based wireless communication systems, which we call TR-TS. In addition, we propose a new ranging method that employs the proposed TR-TS method and a high-resolution ToA estimation technique.

The proposed methods take advantage of the TR technique to convert the errors in time-stamping the packets at the radios into a linear phase rotation across the OFDM subcarriers. TR technique cancels the effect of the channel phase, thus, facilitating the estimation of the linear phase whose slope is proportional to the difference of the OFDM symbol timing errors. Therefore, the TR-TS method achieves high performance even in severe multipath environments regardless whether the channel is minimum, mixed, or maximum phase channel. An algorithm called FFT-WLS algorithm was developed to estimate the linear phase rotation. The CRLB is established and the comparison shows that the MSE of the proposed FFT-WLS attains the CRLB even at low SNR values and small number of observations.

Using the proposed TR-TS method for ranging, all the computations take place at one radio and the second radio can be very simple so that it merely performs TR. The TS and the range estimation between wireless terminals can be performed on demand, without the requirement of frequent message exchange. The results show that by using TR-TS method, lowvariance unbiased range estimates between wireless terminals can be achieved.

Practical aspects such as message delays, carrier frequency offsets, and clock drifts have also been addressed. It is also demonstrated that the proposed method works reliably with real-world measured channels.

\section{APPENDIX}

The noise terms $\tilde{\mathcal{V}}_{n}$ and $\tilde{\mathcal{W}}_{n}$ in (26) are given by

$$
\begin{aligned}
\tilde{\mathcal{V}}_{n}= & \sum_{l=-N_{\mathrm{a}}}^{N_{\mathrm{a}}} \mathcal{V}_{l} \frac{\sin \left[\left(l-n+\epsilon_{A}\right) \pi\right]}{N \sin \left[\left(l-n+\epsilon_{A}\right) \pi / N\right]} \\
& \times \exp \left[j \pi \frac{N-1}{N}\left(l-n+\epsilon_{A}\right)\right] \text { and } \\
\tilde{\mathcal{W}}_{n}^{*}= & \exp \left(j \frac{2 \pi n}{N} \zeta_{A}\right) \sum_{l=-N_{\mathrm{a}}}^{N_{\mathrm{a}}} \mathcal{H}_{l} \frac{\sin \left[\left(l-n-\epsilon_{A}\right) \pi\right]}{N \sin \left[\left(l-n-\epsilon_{A}\right) \pi / N\right]} \\
& \times \sum_{p \neq n}^{N_{\mathrm{a}}} \mathcal{W}_{p}^{*} \frac{\sin \left[\left(p-l+\epsilon_{B}\right) \pi\right]}{N \sin \left[\left(p-l+\epsilon_{B}\right) \pi / N\right]} \\
& \times \exp \left[j \pi \frac{N-1}{N}\left(2 l-p-n+\epsilon_{B}-\epsilon_{A}\right)\right]
\end{aligned}
$$




\section{REFERENCES}

[1] A. Haghparast, T. Abrudan, and V. Koivunen, "OFDM ranging in multipath channels using time-reversal method," in Proc. 10th IEEE Workshop Signal Process. Adv. Wireless Commun., Jun. 2009, pp. $568-572$.

[2] L. Lamport, "Time, clocks, and the ordering of events in a distributed system," Commun. ACM, vol. 21, no. 7, pp. 558-565, Jul. 1978.

[3] IEEE Standard for a Precision Clock Synchronization Protocol for Networked Measurement and Control Systems, IEEE Standard 1588-2008, Mar. 2008.

[4] J. C. Eidson, R. W. Kneifel, A. Moldovansky, and S. P. Woods, "Synchronizing measurement and control systems," Sensors Mag., vol. 19, Nov. 2002.

[5] Y.-C. Wu, Q. Chaudhari, and E. Serpedin, "Clock synchronization of wireless sensor networks," IEEE Signal Process. Mag., vol. 28, no. 1, pp. 124-138, Jan. 2011.

[6] F. Tong and Y. Akaiwa, "Theoretical analysis of interbase-station synchronization systems," IEEE Trans. Commun., vol. 45, no. 5, pp. 590-594, May 1998.

[7] E. Sourour and M. Nakagawa, "Mutual decentralized synchronization for intervehicle communications," IEEE Trans. Veh. Technol., vol. 48, no. 6, pp. 2015-2027, Nov. 1999.

[8] J. N. Lanemann and G. W. Wornell, "Distributed space-time coded protocols for exploiting cooperative diversity in wireless networks," IEEE Trans. Inf. Theory, vol. 49, no. 10, pp. 2415-2425, Oct. 2003.

[9] D. R. Brown and H. V. Poor, "Time-slotted round-trip carrier synchronization for distributed beamforming," IEEE Trans. Signal Process., vol. 56, no. 11, pp. 5630-5643, Nov. 2008.

[10] C. Cormio and K. R. Chowdhury, "A survey on MAC protocols for cognitive radio networks," Ad Hoc Netw., vol. 7, no. 7, pp. 1315-1329, Sep. 2009.

[11] W. Ye, J. Heidemann, and D. Estrin, "Medium access control with coordinated adaptive sleeping for wireless sensor networks," IEEE/ACM Trans. Netw., vol. 12, no. 3, pp. 493-506, Jun. 2004.

[12] H. Liu, H. Darabi, P. Banerjee, and J. Liu, "Survey of wireless indoor positioning techniques and systems," IEEE Trans. Syst., Man, Cybern. Part C, Appl. Rev., vol. 37, no. 6, pp. 1067-1080, Nov. 2007.

[13] D. Wang and M. Fattouche, "OFDM transmission for time-based range estimation," IEEE Signal Process. Lett., vol. 17, no. 6, pp. 571-574, Jun. 2010.

[14] P. Kyritsi, G. Papanicolaou, P. Eggers, and A. Oprea, "Time reversal techniques for wireless communications," in Proc. IEEE 60th Veh. Technol. Conf., vol. 1. Sep. 2007, pp. 47-51.

[15] N. Dharamdial, R. Adve, and R. Farha, "Multipath delay estimation using matrix pencil," Wireless Commun. Netw., vol. 5, pp. 632-635, Mar. 2003

[16] Y. Hua and T. K. Sarkar, "Matrix pencil method for estimating parameters of exponentially damped/undamped sinusoids in noise," IEEE Trans. Acoust., Speech, Signal Process., vol. 38, no. 5, pp. 814-824, May 1990.

[17] L. Song, R. Adve, and D. Hatzinakos, "Matrix pencil for positioning in wireless ad hoc sensor network," in Proc. 1st EWSN, Jan. 2004 pp. $18-27$.

[18] H. Kopetz and W. Schwabl, "Global time in distributed real-time systems," Technische Univ. Wien, Wien, Austria, Tech. Rep. 15/89, 1989.

[19] S. Ganeriwal, R. Kumar, and M. B. Srivastava, "Timing-sync protocol for sensor networks," in Proc. 1st Int. Conf. Embedded Netw. Sensor Syst., Nov. 2003, pp. 138-149.

[20] R. L. Scheiterer, C. Na, D. Obradovic, and G. Steindl, "Synchronization performance of the precision time protocol in industrial automation networks," IEEE Trans. Instrum. Meas., vol. 58, no. 6, pp. 1849-1857, Jun. 2009.

[21] C. N. M. Marins, P. Kaufmann, A. Alves Ferreira, Jr., P. H. Lopes, M. C. de Paiva, R. Mendes Vilela, and A. C. da Silveira, "Precision clock and time transfer on a wireless telecommunication link," IEEE Trans. Instrum. Meas., vol. 59, no. 3, pp. 512-518, Mar. 2010.

[22] B. Sundararaman, U. Buy, and A. D. Kshemkalyani, "Clock synchronization for wireless sensor networks: A survey," Ad Hoc Netw., vol. 3, no. 3, pp. 281-323, May 2005.

[23] F. Sivrikaya and B. Yener, "Time synchronization in sensor networks: A survey," IEEE Netw., vol. 18, no. 4, pp. 45-50, Jul./Aug. 2004.

[24] J.-H. Chiang and T.-C. Chiueh, "Accurate clock synchronization for IEEE 802.11-based multi-hop wireless networks," in Proc. 17th IEEE ICNP, Oct. 2009, pp. 11-20
[25] J. Elson, L. Girod, and D. Estrin, "Fine-grained network time synchronization using reference broadcasts," in Proc. 5th Symp. OSDI, vol. 36. Dec. 2002, pp. 147-163.

[26] Q. Li and D. Rus, "Global clock synchronization in sensor networks," IEEE Trans. Comput., vol. 55, no. 2, pp. 214-226, Feb. 2006.

[27] S. PalChaudhuri, A. Saha, and D. B. Johnson, "Adaptive clock synchronization in sensor networks," in Proc. 3rd Int. Symp. IPSN, Apr. 2004, pp. $340-348$.

[28] K. Römer, "Time synchronization in ad hoc networks," in Proc. 2nd ACM Int. Symp. Mobile Ad Hoc Netw. Comput., Oct. 2001, pp. $173-182$.

[29] O. Simeone, U. Spagnolini, Y. Bar-Ness, and S. Strogatz, "Distributed synchronization in wireless networks," IEEE Signal Process. Magaz., vol. 25 , no. 5, pp. 81-97, Sep. 2008

[30] S. Gezici, Z. Tian, G. B. Giannakis, H. Kobayashi, A. F. Molisch, H. V. Poor, and Z. Sahinoglu, "Localization via ultra-wideband radios: A look at positioning aspects of future sensor networks," IEEE Signal Process. Mag., vol. 22, no. 4, pp. 70-84, Jul. 2005.

[31] Y. Zhou, C. L. Law, Y. L. Guan, and F. Chin, "Indoor elliptical localization based on asynchronous UWB range measurement," IEEE Trans. Instrum. Meas., vol. 60, no. 1, pp. 248-257, Jan. 2011.

[32] T. M. Schmidl and D. C. Cox, "Robust frequency and timing synchronization for OFDM," IEEE Trans. Commun., vol. 45, no. 12, pp. 1613-1621, Dec. 1997.

[33] H. Minn, V. K. Bhargava, and K. Letaief, "A robust timing and frequency synchronization for OFDM systems," IEEE Trans. Wireless Commun., vol. 2, no. 4, pp. 822-839, Jul. 2003.

[34] M. Morelli, C.-C. J. Kuo, and M.-O. Pun, "Synchronization techniques for orthogonal frequency division multiple access (OFDMA): A tutorial review," Proc. IEEE, vol. 95, no. 7, pp. 1394-1427, Jul. 2007.

[35] B. Yang, K. B. Letaief, R. S. Cheng, and Z. Cao, "Timing recovery for OFDM transmission," IEEE J. Sel. Areas Commun., vol. 18, no. 11, pp. 2278-2291, Nov. 2000.

[36] Y. Mostofi and D. C. Cox, "A robust timing synchronization design in OFDM systems-part I: Low-mobility cases," IEEE Trans. Wireless Commun., vol. 6, no. 12, pp. 4329-4339, Dec. 2007.

[37] C. Mensing, S. Plass, and A. Dammann, "Synchronization algorithms for positioning with OFDM communications signals," in Proc. 4th WPNC, Mar. 2007, pp. 205-210.

[38] G. Sun, J. Chen, W. Guo, and K. J. R. Liu, "Signal processing techniques in network-aided positioning: A survey of state-of-theart positioning designs," IEEE Signal Process. Mag., vol. 22, no. 4 , pp. 12-23, Jul. 2005.

[39] F. Gustafsson and F. Gunnarsson, "Mobile positioning using wireless networks: Possibilities and fundamental limitations based on available network measurements," IEEE Signal Process. Mag., vol. 22, no. 4, pp. 41-53, Jul. 2005

[40] C.-Y. Wen, R. D. Morris, and W. A. Sethares, "Distance estimation using bidirectional communications without synchronous clocking," IEEE Trans. Signal Process., vol. 55, no. 5, pp. 1927-1939, May 2007.

[41] X. Li and K. Pahlavan, "Super-resolution TOA estimation with diversity for indoor geolocation," IEEE Trans. Wireless Commun., vol. 3, no. 1, pp. 224-234, Jan. 2004.

[42] A. A. Ali and A. S. Omar, "Time of arrival estimation for WLAN indoor positioning systems using matrix pencil super resolution algorithm," in Proc. 2nd WPNC, Mar. 2005, pp. 11-20.

[43] P. J. Voltz and D. Hernandez, "Maximum likelihood time of arrival estimation for real time physical location tracking of $802.11 \mathrm{a} / \mathrm{g}$ mobile stations in indoor environments," in Proc. Posit. Location Navigat. Symp., Apr. 2004, pp. 585-591.

[44] B. Denis, J. Pierrot, and C. Abou-Rjeily, "Joint distributed synchronization and positioning in UWB ad hoc networks using TOA," IEEE Trans. Microw. Tech., vol. 54, no. 4, pp. 1896-1911, Apr. 2006.

[45] N. Alsindi, X. Li, and K. Pahlavan, "Analysis of time of arrival estimation using wideband measurements of indoor radio propagations," IEEE Trans. Instrum. Meas., vol. 56, no. 5, pp. 1537-1545, Oct. 2007.

[46] J. M. F. Moura and Y. Jin, "Detection by time reversal: Single antenna," IEEE Trans. Signal Process., vol. 55, no. 1, pp. 187-201, Jan. 2007.

[47] T. Shimura, Y. Watanabe, and H. Ochi, "A basic research on the long horizontal active time reversal communication," in Proc. MTTS/IEEE OCEANS, vol. 4. Nov. 2004, pp. 2219-2224.

[48] Z. Yun and M. F. Iskandar, "Time reversal with single antenna systems in indoor multipath environment: Spatial focusing and time compression," in Proc. IEEE Antenna Propag. Soc. Int. Symp., Jul. 2008, pp. 695-698.

[49] E. Panayirci and C. N. Georghiades, "Joint ML timing and phase estimation in OFDM systems using the EM algorithm," in Proc. IEEE ICASSP, vol. 5. Jun. 2000, pp. 2949-2952. 
[50] E. G. Larsson, G. Liu, J. Li, and G. B. Giannakis, "Joint symbol timing and channel estimation for OFDM based WLANs," IEEE Commun. Lett., vol. 5, no. 8, pp. 325-327, Aug. 2001.

[51] C. A. Balanis, Antenna Theory: Analysis and Design, 3rd ed. New York, NY, USA: Wiley, 2005.

[52] Wireless LAN Medium Access Control (MAC) and Physical Layer (PHY) Specifications: High-speed Physical Layer in the $5 \mathrm{GHz}$ Band, IEEE Standard 802.11a 1999.

[53] T. S. Rappaport, Wireless Communications: Principles and Practice, 2nd ed. Englewood Cliffs, NJ, USA: Prentice-Hall, Jan. 2002.

[54] R. Prasad, OFDM for Wireless Communications Systems (Universal Personal Communications). Norwood, MA, USA: Artech House, 2004.

[55] V.-M. Kolmonen, P. Almers, J. Salmi, J. Koivunen, K. Haneda, A. Richter, F. Tufvesson, A. F. Molisch, and P. Vainikainen, "A dynamic dual-link wideband MIMO channel sounder for $5.3 \mathrm{GHz}$," IEEE Trans. Instrum. Meas., vol. 59, no. 4, pp. 873-883, Apr. 2010.

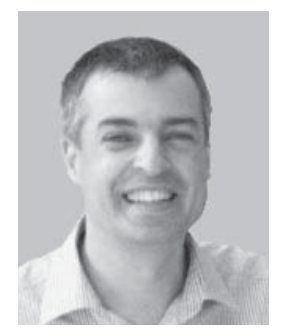

Traian E. Abrudan (S'02-M'09) received the D.Sc. (Hons.) degree from Aalto University, Espoo, Finland (formerly known as Helsinki University of Technology) in 2008, and the M.Sc. degree from the Technical University of Cluj-Napoca, Cluj-Napoca, Romania in 2000 .

He was a member of the SMARAD (SMArt RADios and Wireless Research Unit) Center of Excellence from 2001 to 2010, which has been selected as the Center of Excellence in research by The Academy of Finland. Since September 2010, he has been a Post-Doctoral Researcher with Instituto de Telecomunicações, Faculty of Engineering, University of Porto, Porto, Portugal. Currently, he has been designing and implementing indoor positioning algorithms using wireless sensor networks. His current research interests include parameter estimation, numerical optimization, sensor array signal processing, and multicarrier communications.
Azadeh Haghparast received the B.Sc. degree in electrical engineering from the Iran University of Science and Technology, Tehran, Iran, in 2002, and the M.Sc. degree in communications engineering from the Helsinki University of Technology, Helsinki, Finland, in 2006. She pursued Doctoral studies in signal processing at Aalto University, Espoo, Finland until 2011.

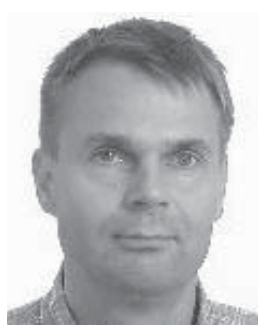

Visa Koivunen (S'87-M'93-SM'98-F'10) received the D.Sc. (Hons.) degree in electrical engineering from the Department of Electrical Engineering, University of Oulu, Oulu, Finland.

He was a Visiting Researcher with the University of Pennsylvania, Philadelphia, PA, USA, from 1992 to 1995 . Since 1999, he has been a Professor of signal processing with Aalto University, Espoo, Finland, (formerly known as Helsinki Univ of Technology). Since 2009, he has been an Academy Professor with Aalto University. He is one of the Principal Investigators in the SMARAD (SMArt RADios and Wireless Research Unit) Center of Excellence nominated by the Academy of Finland. He was an Adjunct Full Professor with the University of Pennsylvania. From 2006 to 2007, he was a Visiting Fellow with Nokia Research Center, Sunnyvale, CA, USA, as well as Princeton University, Princeton, NJ, USA. He makes frequent research visits to Princeton University. He has published more than 350 papers in international scientific conferences and journals. His current research interests include statistical, communications, radar, and sensor array signal processing.

Dr. Koivunen received the Primus Doctor (Best Graduate) Award among the doctoral graduates from 1989 to 1994 . He is a member of Eta Kappa $\mathrm{Nu}$. He has co-authored papers which received the Best Paper Award in IEEE PIMRC in 2005, EUSIPCO in 2006, and EuCAP in 2006. He received the IEEE Signal Processing Society Best Paper Award in 2007 (co-authored with J. Eriksson). He is an Editorial Board Member for the IEEE Signal Processing Magazine. He is a member of the IEEE Sensor Array Multichannel Signal Processing Technical Committee. He is serving at the Industrial Liaison Board of the IEEE SP society. He was the General Chair of the IEEE Signal Processing Advances in Wireless Communication Conference, Helsinki, in 2007. 